\title{
Mass balance errors in modeling two-phase immiscible flows: causes and remedies
}

\author{
Linda M. Abriola \& Kaus Rathfelder \\ Environmental and Water Resources Engineering, Department of Civil and Environmental Engineering, The Liniversity of Michigan, \\ Ann Arbor, Michigan 48109-2125. USA
}

\begin{abstract}
Mass balance accuracy of two-phase immiscible flow models used for contaminant hydrology applications is examined through comparisons of finite element and finite difference solutions of the pressure-based and pressure-saturation formulations. The influence of model formulation and initial conditions on mass balance performance is explored. Model simulations demonstrate that accurate solutions for multiphase flow problems can be obtained with either finite element or finite difference, pressure-based or pressure-saturation formulations, if coefficients and initial conditions are properly treated. In the pressure-based formulation, capacity coefficients arise from the expansion of the saturation variables in terms of capillary pressure. Mass balance accuracy depends upon the proper evaluation of the capacity coefficients when the capillary pressure-saturation relation is nonlinear. Capacity coefficient approximations for finite element pressure-based models are developed which preserve elemental expansion of the saturation derivative. These approximations are shown to produce good mass balance results and accurate solutions, in contrast with uraditional finite element approaches. When the organic liquid is initially absent from a domain, simulations reveal that mass balance accuracy is obtained only when the initial pressure distribution is established from a zero capillary pressure condition. The influence of matrix mass lumping and the minimum value of the capacity coefficient on model performance is also investigated.
\end{abstract}

Key words: NAPL, immiscible, multiphase, flow, numerical model, mass balance.

\section{NOTATION}

$C_{\alpha}=\frac{\partial S_{a}}{\partial P_{c}}$

$\boldsymbol{F}_{\boldsymbol{\alpha}}$

$\boldsymbol{k}$

$\boldsymbol{k}_{\mathrm{ra}}$

$L_{e}$

$M_{\alpha}$

$m, n, \alpha$

N

$P_{\alpha}$

$P_{c}=P_{o}-P_{n}$

$S_{\alpha}, \bar{S}_{\alpha}, S_{r \alpha}$

$\boldsymbol{t}$ $a$-fluid capacity coefficient

a-fluid capacity coeficient approximations by the analytical or scs methods $a$-fluid mass transport across the computational domain intrinsic permeability tensor $\alpha$-fluid relative permeability length of the linear elements $a$-fluid mass storage

fitting parameters of the van Genuchten fitting function

linear basis functions

$\alpha$-fiuid pressure

capillary pressure

$a$-fluid saturation, effective saturation, and residual saturation time

Advances in Water Resources 0309-1708;93/506.00

C 1993 Elsevier Science Publishers Ltd. $\boldsymbol{w}$

$z$

$3_{a}, 3_{m}$

$\Delta$,

$\Delta z, \Delta l$

$\boldsymbol{\epsilon}$

$\epsilon_{\alpha}$

$\gamma_{a}$

$\lambda_{\alpha}=\frac{k_{k a}}{\mu_{\alpha}}$

$\mu_{\alpha}$

$\bar{\phi}, 0^{0}$

$\rho_{a}$

$[A]^{e}$

$\left[A^{\prime}\right]^{e}$

$\left.{ }^{-B}\right]^{e}$
$\boldsymbol{F}^{e}$ time weighting coefficient for the analytical $C_{\alpha}$ approximations vertical distance

fluid and matrix compressibilities backward difference operator spatial and temporal discretization convergence criterion elemental residual between the discretized temporal saturation derivative and the expanded form

$\alpha$-fluid specific weight

$\alpha$-fluid transmissibility

$\alpha$-fluid dynamic viscosity

porosity, porosity at the reference pressure

$a$-fluid density

local mass matrix

local mass matrix obtained in the FEcs

formulation

local stiffness matrix

local right hand side matrix 


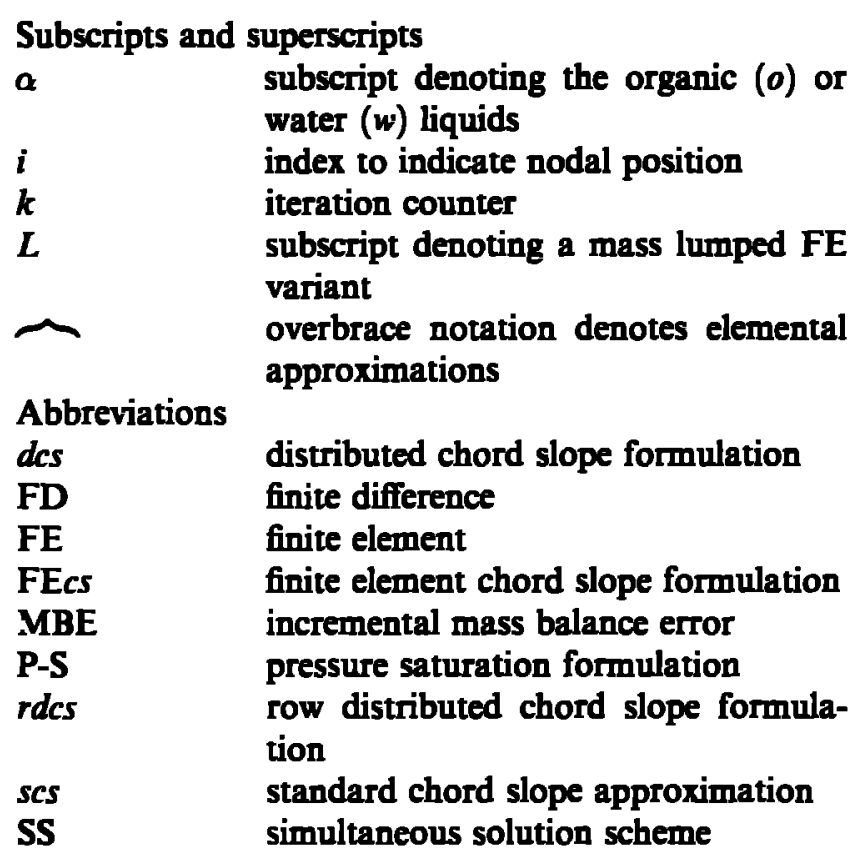

\section{INTRODLCTION}

Widespread subsurface contamination by hazardous organic non-aqueous phase liquids (NAPLs) has focused attention on the development of predictive models for immiscible multiphase fluid flow. Although several mathematical modeling strategies have been employed, the most attractive approaches in terms of accuracy and flexibility are multiphase flow models based upon the solution of coupled mass balance equations. ${ }^{2,5,25}$ The majority of immiscible flow simulators in the contaminant hydrology literature employ implicit time-stepping procedures in the simultaneous solution (SS) of the phase mass balance equations. ${ }^{2-4,9-12,17.19,20,24,27,28}$ Depending upon the selection of the primary dependent variables in the SS scheme, alternative formulations can be developed. Two approaches examined in this work are the pressure-based formulation and a mixed pressure-saturation (P-S) formulation.

The pressure-based formulation uses fluid pressures as the primary variables. These state variables are continuous, regardless of soil heterogeneity or liquid saturation distributions. Two-dimensional pressurebased models have been developed with finite difference $(F D)^{3,4}$ and finite element (FE) solution procedures. ${ }^{17,20,24,27,28}$ Alternatively, the P-S formulation employs water saturation and water or organic pressure as the primary variables. Air pressure remains static or is not considered in models developed to date. The P-S formulation has been implemented with FD methods in two ${ }^{9.19}$ and three dimensions. ${ }^{10}$

A crucial step in the construction of the pressurebased formulation is the expansion of the saturation derivative in terms of pressure, incorporating saturation variables into the so-called capacity coefficient. Material balance accuracy is known to be sensitive to the specification of this capacity coefficient. FD models employing the standard chord slope approximation of the capacity coefficient have been shown to conserve mass for applications in petroleum engineering, ${ }^{6}$ soil science, ${ }^{7,23}$ and contaminant hydrology. ${ }^{3,4}$ However, several researchers have reported poor mass balance accuracy in FE pressure-based models with applications in soil science ${ }^{7.23}$ and contaminant hydrology. ${ }^{17}$ Efforts to remedy these errors have focused on the procedure used to evaluate the capacity coefficient. ${ }^{17}$ Numerical experiments have produced mixed success, however, and a comprehensive explanation of the errors has not been presented. In one instance the pressure-based formulation was abandoned entirely in favor of an alternative mass conservative formulation which did not employ capacity coefficients.'

Proponents of the P-S formulation have reported good mass balance accuracy for this method., ${ }^{9,19}$ All P-S models described to date consider a restricted case involving two-phase flow of incompressible fluids in a rigid soil matrix. Such a scenario does not require the use of capacity coefficients, eliminating a source of mass balance errors observed in pressure-based models. When three phase flow or compressibility effects are considered, however, the P-S formulation will incorporate capacity coefficients. L'nder such conditions, mass balance problems observed with the pressure-based models may also become important in the P-S formulation.

The ability of a numerical model to conserve mass is a necessary, but not sufficient, condition for solution accuracy. A model which exhibits poor mass balance behaviour has limited practicality because of uncertainties in the model predictions. The need for accurate mass conservation in generalized multiphase flow models has motivated the research presented herein. The objectives of this work are to identify potential sources of mass balance errors in FE and FD multiphase flow models, to elucidate the causes of these errors, and to propose and evaluate approaches which can remedy such problems. For simplicity, model comparisons are restricted to one-dimensional, two-phase flow scenarios. Four aspects of a numerical model which may impact mass balance accuracy are examined: the specification of the initial pressure distribution, the evaluation method for the capacity coefficient, the assignment of a minimum capacity coefficient, and mass lumping procedures.

\section{NUMERICAL PROCEDLRES}

\section{Governing equations}

The conventional form of the fluid phase mass 
balance equations for a two phase organic-water system is:

$$
\frac{\partial}{\partial t}\left[\rho \rho_{\alpha} S_{\alpha} \bar{i}=\nabla \cdot \rho_{\alpha} \lambda_{\alpha}\left(\nabla P_{\alpha}-\gamma_{\alpha} \nabla z\right)\right. \text {. }
$$

where $\alpha=w, o$ repesent the fluid phases ( $w=$ water, $o=$ NAPL), $t$ is time [T], $o$ is the dimensionless porosity, $S_{\alpha}$ is the dimensionless $\alpha$-fluid saturation, $\rho_{\alpha}$ is the $\alpha$ fluid density $M / L^{3-}, P_{\alpha}$ is $\alpha$-fluid pressure $\left.M / L T^{2}\right], \gamma_{\alpha}$ is the $\alpha$-fluid specific weight $M / L^{2} T^{2} \mathrm{j}, z$ is the positive downward vertical direction, and

$$
\lambda_{\alpha}=\frac{k k_{r \alpha}}{\mu_{\alpha}}
$$

is the $\alpha$-fluid transmissibility, where $k$ is the intrinsic permeability tensor $L^{2-}, k_{r \alpha}$ is the dimensionless $\alpha$-fluid relative permeability, and $\mu_{\alpha}$ is the $\alpha$-fluid dynamic viscosity $\left[M / L T^{-}\right.$. Auxiliary relations include:

1. Continuity of fluid saturations and pore volume,

$$
S_{w}-S_{o}=1
$$

2. Capillary pressure-saturation relationships, represented in this study by the functional form of van Genuchten, ${ }^{29}$

$$
\bar{S}_{w}=\frac{S_{m}-S_{m}}{1-S_{m}}=\left[\frac{1}{1-\left(\alpha P_{c}\right)^{n}}\right]^{m}
$$

where

$$
P_{c}=P_{o}-P_{w}
$$

is the capillary pressure between the organic and aqueous phases, $S_{m}$ is the irreducible water saturation, $\bar{S}_{k}$ is the effective water saturation, and $\alpha, n$, and $m=1-1 / n$ are fitting parameters.

3. Relative permeability - saturation relationships, ${ }^{29}$

$$
\begin{aligned}
k_{r i} & \left.=\left(\bar{S}_{m}\right)^{1 / 2-1}-\left(1-\bar{S}_{m}^{1 / m}\right)^{m}\right]^{2} \\
k_{r o} & =\left(1-\bar{S}_{w}\right)^{1 / 2}\left[1-\bar{S}_{w}^{1 / m}\right]^{2 m}
\end{aligned}
$$

Assumptions in this two-phase immiscible flow formulation include negligible interphase mass transfer, no internal source sinks, and unique functional relations for $P_{c}\left(S_{w}\right)$ and $k_{r}\left(S_{w}\right)$, ignoring hysteresis and organic liquid entrapment.

\section{Simultaneous solution formulations}

The pressure-based SS scheme recasts the phase mass balance eq (1) in terms of the selected primary dependent variables, fluid pressures. Expanding the accumulation derivatives in terms of capillary pressure, eq (1) can be rearranged as:

$$
\begin{aligned}
& \Theta \rho_{\alpha} C_{\alpha}\left[\frac{\partial P_{o}}{\partial t}-\frac{\partial P_{\alpha}}{\partial t}\right]+\phi \rho_{\alpha} S_{\alpha} \Theta_{\alpha} \frac{\partial P_{\alpha}}{\partial t} \\
& +\frac{1}{2} \rho_{\alpha} S_{\alpha} \Theta^{\sigma} 3_{m}\left[\frac{\partial P_{\sigma}}{\partial t}+\frac{\partial P_{u}}{\partial t}\right]=\nabla \cdot\left[\lambda_{\alpha}\left(\nabla P_{\alpha}-\gamma_{\alpha} \nabla z\right)\right]
\end{aligned}
$$

where $3_{\alpha}$ and $3_{m}$ are the $\alpha$-fluid and matrix compressibilities, $\rho^{\circ}$ is the porosity at the reference pressure, and

$$
C_{\alpha}=\frac{\partial S_{\alpha}}{\partial P_{c}}
$$

is the $\alpha$-fluid capacity coefficient. The pressure-based formulation easily accommodates the matrix and fluid compressibility effects, although in typical contaminant hydrology applications these are often considered minor and are neglected. $9,10,17,19,20,24$ In this work compressibility effects are neglected to facilitate comparisons with a P-S model.

P-S formulations developed for contaminant hydrology problems employ water saturation and either organic or water pressure as the primary dependent variables. Models developed to date also neglect fluid and matrix compressibilities in order to completely eliminate the capacity-type coefficients. Following the approach of Faust, ${ }^{9}$ the mass balance equations for a rigid matrix and incompressible fluids can be rearranged using eqns (3) and (5) to eliminate $S_{c}$ and $P_{w}$ :

$$
\begin{aligned}
o \frac{\partial S_{w}}{\partial t} & \left.\left.=\nabla \cdot \lambda_{w}\left(\nabla P_{o}-\nabla P_{c}\right)-\gamma_{w} \nabla z\right)\right] \\
-\odot \frac{\partial S_{w}}{\partial t} & =\nabla \cdot \lambda_{o}\left(\nabla P_{\sigma}-\tau_{o} \nabla z\right)_{-}^{-}
\end{aligned}
$$

This P-S formulation is considered well-suited to conditions where $S_{o}=0$ because $S_{o}$ does not appear explicitly in eqns (10) or (11). ${ }^{19}$

\section{Numerical models}

The pressure-based formulation, eq (8), was solved with FD and FE algorithms. FD solutions were obtained with the model of Abriola, ${ }^{3,4}$ modified for two-phase immiscible flow. Considering only the vertical direction, the FD analogs employed in this model are:

$$
\begin{aligned}
o C_{\alpha_{1}} \Delta_{t} P_{c_{1}}= & \frac{1}{2(\Delta z)^{2}}-\lambda_{t-\frac{i}{2}} P_{\alpha_{1-}}: \\
& -\left(\lambda_{i-\frac{i}{2}}-\lambda_{t-\frac{i}{2}}\right) P_{i}-\lambda_{i-\frac{i}{2}} P_{\alpha_{i}-1}- \\
& +\frac{1}{\Delta z} \gamma_{\alpha_{i}}\left(\lambda_{t-\frac{i}{2}}+\lambda_{i-\frac{i}{2}}\right)
\end{aligned}
$$

where $\Delta_{t}$ is the backward difference operator in time and $\Delta z$ is the uniform spacing of block $i$ in the discretized space domain. The interblock transmissibility terms are evaluated as arithmetic means to ensure correspondence with FE solutions.

Pressure-based FE solutions were obtained with the model of Reeves \& Abriola. ${ }^{27,28}$ This model uses standard piecewise linear interpolating functions to approximate elemental pressure, mobility and capacity variables,

$$
\widehat{P}_{a}(z, t) \simeq \sum_{i=1}^{2} P_{a_{1}}(t) N_{i}(z)
$$




$$
\begin{aligned}
& \hat{\lambda}_{\alpha}(z, t) \simeq \sum_{i=1}^{2} \lambda_{\alpha_{i}}(t) N_{i}(z) \\
& \hat{C}_{\alpha}(z, t) \simeq \sum_{i=1}^{2} C_{\alpha_{1}}(t) N_{i}(z)
\end{aligned}
$$

where $N_{i}$ are the linear basis functions. Applying the Galerkin finite element method in local coordinates yields the elemental equations:

$$
\left.A_{\cdot}^{i e}\left\{\begin{array}{l}
\Delta_{1} P_{c_{*}} \\
\Delta_{l} P_{c_{2}}
\end{array}\right\}=[B]^{e}\left\{\begin{array}{l}
P_{\alpha_{:}} \\
P_{\alpha 2}
\end{array}\right\}-: F\right]^{e}
$$

where subscripts 1 and 2 refer to the left and right nodes of the linear elements, respectively, $L_{\varepsilon}$ is the length of the element, and the elemental matrices are:

$$
\begin{aligned}
& {\left[A_{-}^{-e}=\frac{o L_{e}}{12}\left[\begin{array}{ll}
3 C_{\alpha 1}+C_{\alpha 2} & C_{\alpha 1}-C_{\alpha 2} \\
C_{\alpha 1}+C_{\alpha 2} & C_{\alpha 1}-3 C_{\alpha 2}
\end{array}\right]\right.} \\
& B_{-}^{\cdot e}=\frac{1}{2 L_{e}}\left[\begin{array}{ll}
-\lambda_{\alpha 1}-\lambda_{\alpha 2} & \lambda_{\alpha 1}-\lambda_{\alpha 2} \\
\lambda_{\alpha 1}+\lambda_{\alpha 2} & -\lambda_{\alpha 1}-\lambda_{\alpha 2}
\end{array}\right] \\
& F !^{e}=\frac{1}{2} \gamma_{\alpha \alpha}\left[\begin{array}{c}
\lambda_{\alpha 1}+\lambda_{\alpha 2} \\
-\lambda_{\alpha 1}-\lambda_{\alpha 2}
\end{array}\right]
\end{aligned}
$$

Because all models evaluate interblock transmissibility coefficients as arithmetic means, the spatial descretization terms in the FD and FE pressure-based models can be shown to be identical. Differences in the solutions of these two models are thus attributable to the temporal discretization terms. ${ }^{7}$

A variant in construction of the mass matrix, $[A]^{e}$, is a diagonalization procedure known as mass 'lumping' 16 yielding,

$$
A]^{e L}=\frac{o L_{e}}{6}\left[\begin{array}{cc}
2 C_{\alpha 1}+C_{\alpha 2} & 0 \\
0 & C_{\alpha 1}-2 C_{\alpha 2}
\end{array}\right]
$$

Mass lumping procedures generally improve numerical stability and minimize oscillatory behavior. Although lumped formulations have been considered superior to the consistent form in applications of unsaturated flow, ${ }^{7,22}$ they have not been broadly applied in multiphase flow models.

A third model was developed which implements the P-S formulation. This model solves the mass balance eqns (10) and (11) using a point-distributed FD scheme. The formulation is identical to that of Faust, ${ }^{9}$ except interblock transmissibility coefficients are evaluated with arithmeric means to ensure compatibility with the pressure-based models.

All numerical models require an iterative solution algorithm due to nonlinearity of the transmissibility and capacity relations. The Newton-Raphson iteration scheme is employed in both FD models, and a Picard interation approach is used in the FE code. A maximum difference convergence criterion is used in all models:

$$
\max \frac{{ }_{\alpha}^{k-1}-P_{\alpha}^{k-}}{P_{\alpha}^{k-1} \mid} \leq \epsilon
$$

where $k$ is the iteration counter and $\epsilon=1(-4)$, unless indicated otherwise.

\section{Capadty coefiicients}

The method used to evaluate $C_{a}$ directly affects mass balance accuracy of the numerical model. Soil scientists and petroleum engineers have recognized that to achieve mass conservation in FD simulators, expansion of the saturation time derivative must be exact, i.e.

$$
\frac{\partial S_{\alpha}}{\partial t}=\frac{\partial S_{\alpha}}{\partial P_{c}} \frac{\partial P_{c}}{\partial t}
$$

The FD analog of eqn (22) leads to the standard chord slope (scs) approximation at node $i$.

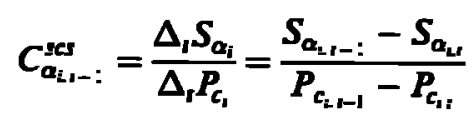

Finite difference schemes employing $C^{\text {scs }}$ produce good mass balance accuracy. ${ }^{6.7 .23}$ In contrast, traditional FE schemes using $C^{s c s}$ demonstrate poor mass balance behavior. ${ }^{7.1722}$

Attempts to improve mass balance accuracy in FE models has motivated various modifications in the evaluation of the capacity coefficient. Kaluarachchi \& Parker ${ }^{17}$ compared a number of procedures based on analytical derivatives of the capillary pressure function, eqn (4). Several of these approximations derive from a weighted average of the analytical derivatives evaluated at the current and previous time levels,

$$
C_{\alpha}^{*}=w^{*} C_{\alpha}\left(P_{c_{i}-1}^{k+1}\right)+1-w_{-}^{*} C_{\alpha}\left(P_{c_{1}}\right)
$$

where weights $w=0.0,0.5$, and 1.0 were tested. Kaluarachchi \& Parker $^{17}$ also examined a mean pressure analytic scheme credited to Osborne \& Sykes. ${ }^{24}$ This approach evaluates the analytical derivative as a function of the time averaged $P_{c}$,

$$
C_{\alpha}^{*}=C_{\alpha}\left(P_{c_{1-1}: 2}\right)
$$

where $P_{c_{1-1 ; 2}}=\frac{1}{2}\left(P_{c_{1-1}}+P_{c_{1}}\right)$.

None of the above analytical schemes was found to be completely adequare in terms of mass balance accuracy and these were subsequently abandoned in favor of a modified chord slope approximation. ${ }^{17}$ This approach is based on an alternative definition of the capacity term, $C_{p q}=\partial S_{p} / \partial P_{q}$, and is similar to the scs method except individual pressure terms are averaged over time. The modified chord slope approximation afforded some improvement in mass balance aceuracy, however errors exceeding 5\% were reported.

Analogous to the derivation of the standard chord slope approximation used in FD solutions, the capacity 
coefficient in FE solutions can be obtained in a manner which ensures that the expansion of $\partial S / \partial t$ is exact. Based on this principle, Milly ${ }^{22}$ developed expressions for the capacity terms in an unsaturated flow model, in conjunction with mass lumping procedures. Following a similar approach, new mass-conservative time-stepping procedures are developed for FE pressure-based models. These procedures are applicable for the development of consistent or lumped mass matrices.

In a traditional FE implementation of the pressurebased formulation, eqns (13) and (15) are employed to separately interpolate $P_{c}$ and $C_{\alpha}$ over each element. Within this traditional framework, the elemental approximation of the saturation derivative in eqn (22) can be equated with the expanded form,

$$
\begin{aligned}
& {\widehat{\partial S_{\alpha}}}_{\partial t} \simeq \sum_{i=1}^{2} \frac{\partial S_{\alpha_{i}}}{\partial t} N_{i} \\
& =\left(\sum_{i=1}^{2} C_{\alpha_{i}} N_{i}\right)\left(\sum_{i=1}^{2} \frac{\partial P_{c_{1}}}{\partial t} N_{i}\right) \simeq \widetilde{C_{\alpha}} \frac{\partial P_{c}}{\partial t}
\end{aligned}
$$

and application of the linear Galerkin finite element method yields

$$
\left.\frac{\oplus L_{e}}{3}\left[\begin{array}{ll}
1 & \frac{1}{2} \\
\frac{1}{2} & 1
\end{array}\right]\left\{\begin{array}{l}
\Delta_{l} S_{\alpha_{1}} \\
\Delta_{l} S_{\alpha_{2}}
\end{array}\right\}=; A\right]^{e}\left\{\begin{array}{l}
\Delta_{l} P_{c_{1}} \\
\Delta_{t} P_{c_{2}}
\end{array}\right\}
$$

Expansion of $\partial S / \partial t$ can be preserved by developing element-dependent capacity coefficients such that the equality in eqn (27) is satisfied. Two procedures were tested. The first method solves the two by two system directly for the unknown element-dependent capacity coefficients producing:

$$
\begin{aligned}
& C_{\alpha_{1}}^{d c s}=\frac{\Delta_{t} S_{\alpha_{1}}\left(\Delta_{t} P_{c_{1}}+5 \Delta_{l} P_{c_{2}}\right)-\Delta_{t} S_{\alpha_{2}}\left(\Delta_{l} P_{c_{2}}-\Delta_{t} P_{c_{1}}\right)}{\left(\Delta_{t} P_{c_{1}}\right)^{2}+4 \Delta_{l} P_{c_{1}} \Delta_{t} P_{c_{2}}+\left(\Delta_{l} P_{c_{2}}\right)^{2}} \\
& C_{\alpha_{2}}^{d c s}=\frac{\Delta_{l} S_{\alpha:}\left(\Delta_{l} P_{c_{1}}-\Delta_{l} P_{c_{2}}\right)+\Delta_{l} S_{\alpha_{2}}\left(5 \Delta_{l} P_{C_{1}}+\Delta_{l} P_{c_{2}}\right)}{\left(\Delta_{l} P_{c_{1}}\right)^{2}+4 \Delta_{l} P_{c_{2}}-\left(\Delta_{l} P_{c_{2}}\right)^{2}}
\end{aligned}
$$

These expressions are referred to herein as distributed chord slope (dcs) approximations because nodal capacity coefficients depend on capillary pressures at both nodes in the element. These approximations were successfully used in a two-phase model developed by Gamliel. 13,14

The second approach treats each equation in (27) separately, developing $C_{a}$ approximations that depend on the row of the local matrix. For row 1 ,

$$
C_{\alpha_{11}}^{\text {rdes }}=\frac{4 \Delta_{t} S_{\alpha_{1}}}{3 \Delta_{l} P_{c_{1}}+\Delta_{t} P_{c_{2}}} \quad C_{\alpha_{12}}^{\text {rdes }}=\frac{2 \Delta_{t} S_{\alpha_{:}}}{\Delta_{l} P_{c_{1}}+\Delta_{l} P_{c_{2}}}
$$

and row 2 ,

$$
C_{\alpha_{i:}:}^{\text {rdes }}=\frac{2 \Delta_{l} S_{\alpha_{2}}}{\Delta_{l} P_{c:}+\Delta_{1} P_{c_{2}}} \quad C_{\alpha_{z z}}^{\text {rdes }}=\frac{4 \Delta_{l} S_{\alpha_{2}}}{\Delta_{1} P_{c:}+3 \Delta_{1} P_{c_{2}}}
$$

where the numbered subscripts are the row and node of the local matrix, respectively. These expressions were developed in the work of Reeves ${ }^{26.28}$ and are termed row distributed chord slope (rdcs) approximations. Both $C_{\alpha}^{\text {des }}$ and $C_{\alpha}^{\text {rdes }}$ yield identities when substituted into eqn (27), ensuring that expansion of $\partial S / \partial t$ is exact over the element.

The des and rdcs approximations can also be developed in conjunction with mass lumping procedures. Expansion of the saturation derivative is preserved in this case by equating the elemental approximations:

$$
\frac{\dot{\theta} L_{e}}{2}\left[\begin{array}{ll}
1 & 0 \\
0 & 1
\end{array}\right]\left\{\begin{array}{l}
\Delta_{l} S_{\alpha_{:}} \\
\Delta_{l} S_{\alpha_{2}}
\end{array}\right\}=\left[A_{j}^{; e_{L}}\left\{\begin{array}{l}
\Delta_{l} P_{c:} \\
\Delta_{l} P_{c_{2}}
\end{array}\right\}\right.
$$

which yields the dcs approximations:

$$
\begin{aligned}
& C_{\alpha_{1}}^{\text {des }_{L}}=\frac{2 \Delta_{t} S_{a_{1}}}{\Delta_{1} P_{c_{:}}}-\frac{\Delta_{t} S_{a_{2}}}{\Delta_{t} P_{c_{2}}} \\
& C_{\alpha_{2}}^{d e_{L}}=\frac{2 \Delta_{t} S_{a_{2}}}{\Delta_{1} P_{c_{2}}}-\frac{\Delta_{t} S_{a_{:}}}{\Delta_{t} P_{c:}}
\end{aligned}
$$

and the rdcs approximations:

$$
\begin{array}{ll}
C_{\alpha_{:}}^{\text {rdcs }}=\frac{3 \Delta_{t} S_{\alpha_{1}}}{2 \Delta_{t} P_{c_{1}}} & C_{\alpha_{12}}^{\text {rddos }}=0 \\
C_{\alpha_{21}}^{\text {rdss }}=0 & C_{\alpha_{22}}^{\text {rdcs }}=\frac{3 \Delta_{t} S_{\alpha_{2}}}{2 \Delta_{t} P_{c_{2}}}
\end{array}
$$

A third approach which preserves expansion of $\partial S / \partial t$ employs a non-traditional FE procedure. Rather than separately interpolating $C_{\alpha}$ and $P_{c}$, an elemental approximation of eqn (22) is defined by collectively interpolating the expanded saturation derivative,

$$
\widehat{\frac{\partial S_{\alpha}}{\partial t}} \simeq \sum_{i=1}^{2} \frac{\partial S_{\alpha_{i}}}{\partial t} N_{i}=\sum_{i=1}^{2} C_{\alpha_{1}} \frac{\partial P_{c_{i}}}{\partial t} N_{i} \simeq \widetilde{C_{\alpha} \frac{\partial P_{c}}{\partial t}}
$$

Applying the Galerkin finite element method to these non-traditional approximations generates eqn (16) with the mass matrix:

$$
\left[A^{\prime}\right]^{e}=\frac{\phi L_{e}}{3}\left[\begin{array}{ll}
C_{\alpha_{1}} & \frac{C_{\alpha_{2}}}{2} \\
\frac{C_{\alpha_{1}}}{2} & C_{a_{2}}
\end{array}\right]
$$

Again equating these approximations with the saturation derivatives in eqn (27) and solving for the capacity coefficients yields the scs approximation in eqn (23). The capacity coefficient approximations in this case are continuous at the elemental boundaries. This formulation is referred to as the FEcs approximation. 


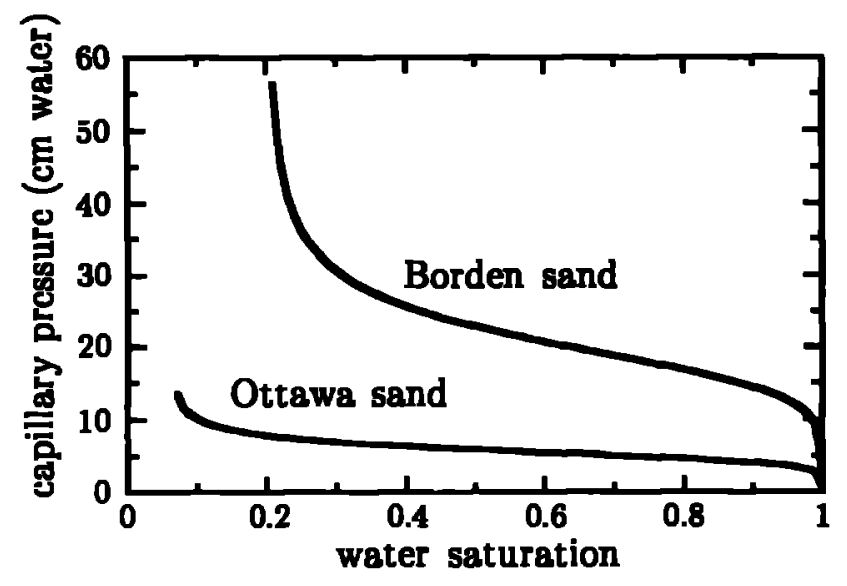

Fig. 1. Drainage $P_{c}\left(S_{w}\right)$ functions.

The mass lumped variant of the FEcs scheme is

$$
A^{\prime} I^{e L}=\frac{o L_{e}}{2}\left[\begin{array}{cc}
C_{\alpha_{:}} & 0 \\
0 & C_{a_{2}}
\end{array}\right]
$$

which is equivalent to the L2 lumping scheme of Milly. ${ }^{22}$ Conceptually, however, this formuation is distinguished as a standard lumping procedure applied to the elemental approximation $\overbrace{S_{\alpha} / \partial t}=\overbrace{C_{\alpha} \partial P_{c} / \partial t}$. Also note that assemblage of this approximation is equivalent to the fully implicit FD approximation. Consequently, evaluation of the capacity coefficients again yields the scs approximation in eqn (23) which is known to produce good mass balance accuracy in FD simulators.

All modified chord slope approximations are easily implemented by substitution into the local matrix. One difficulty arises with chord slope approximations when the time rate of change in $P_{c}$ is small, such that the denominator approaches zero. In this work, when implementing any of the chord slope approximations, the absolute value of the denominator was inspected and required to be greater than $1(-10)$, otherwise $C_{\alpha}$ was evaluated with an analytical derivative of $P_{c}\left(S_{w}\right)$.
Additionally, $C_{\alpha}$ was assigned a minimum absolute value of $1(-6)$ whenever calculated values fell below this level. The consequence of using a nonzero minimum $C_{\alpha}$ is examined in a later section.

\section{NLMERICAL EXPERIMENTS AYD ANALYSIS}

\section{Simulation conditions}

The following numerical simulations involve onedimensional vertical two-phase flow in a homogeneous porous medium. All solutions were obtained on a uniform mesh with variable time increments. The time step sizes were adjusted dynamically based on the number of iterations required for convergence but were restricted to a specified maximum size.

The influence of soil and fluid properties on numerical mass balance behavior was examined with comparisons from two media contaminant systems. Transport of trichloroethane in Borden sand was simulated with data from Demond \& Roberts. ${ }^{8}$ The second system employs data from Kueper et al. ${ }^{18}$ and Høst-Madsen, ${ }^{15}$ simulating the migration of tetrachloroethylene in Ottawa sand. The Ottawa sand is a uniform, permeable soil that is readily drained over small displacement pressures. Conversely, the well-sorted Borden sand is less permeable and has a wider pore distribution, resulting in larger displacement pressures. All soil and fluid properties are listed in Table 1 and $P_{c}\left(S_{2}\right)$ relations for both systems are shown in Fig. 1.

\section{Mass balance calculation}

Mass balance checks were used to assess each simulator's ability to solve the governing flow equations, providing a necessary but not sufficient measure of solution accuracy. An incremental mass balance

Table 1. Soll and fiuid parameters ased in simulations

\begin{tabular}{|c|c|c|}
\hline Parameter & System 1 & System 2 \\
\hline $\begin{array}{l}\text { Soil type } \\
\text { Porosity } \\
\text { Intrinsic permeability }\left(\mathrm{cm}^{2}\right) \\
\text { Matrix compressibility }\left(\mathrm{cm}^{2} \text {.dyne) }\right. \\
\text { Residual water saturation } \\
\text { van Genuchten fitting parameters }\end{array}$ & $\begin{array}{l}\text { Borden sand } \\
0.33 \\
8.36 \times 10^{-3} \\
0.0 \\
0.204\end{array}$ & $\begin{array}{l} \pm 25 \text { Ottawa sand } \\
0.39 \\
2.05 \times 10^{-6} \\
0.0 \\
0.0617\end{array}$ \\
\hline$a\left(\mathrm{~cm}^{2}\right.$ dyne $)$ & $\begin{array}{l}5.2 \times 10^{-5} \\
5.62\end{array}$ & $\begin{array}{l}1.87 \\
6.19\end{array}$ \\
\hline $\begin{array}{l}\text { Organic contaminant } \\
\text { molecular weight ( } \mathrm{g} \mathrm{mol} \text { ) } \\
\text { Density (g.cm) } \\
\text { Viscosity (poise) } \\
\text { Compressibility (cm }{ }^{2} \text { dyne) }\end{array}$ & $\begin{array}{c}\text { 1,1,2-trichloroethane } \\
133.4 \\
1.44 \\
0.0119 \\
0.0\end{array}$ & $\begin{array}{l}\text { tetrachloroethylene } \\
143.8 \\
1.61 \\
0.009 \\
0.0\end{array}$ \\
\hline 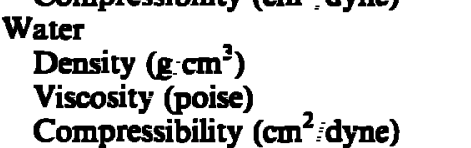 & $\begin{array}{l}1.0 \\
0.01 \\
0.0\end{array}$ & $\begin{array}{l}1.0 \\
0.01 \\
0.0\end{array}$ \\
\hline
\end{tabular}


coefficient was calculated at each time step using

$$
\mathrm{MBE}_{\alpha}=\frac{M_{\alpha}^{\prime-\Delta t}-M_{\alpha}^{t}}{F_{\alpha}^{\Delta t}}
$$

where $M_{\alpha}^{t}$ is the $\alpha$-fluid mass storage at time $t$ and $F_{\alpha}^{\Delta r}$ is the net $\alpha$-fluid mass entering the computation domain during the time step $\Delta t$. An $\mathrm{MBE}_{\alpha}$ value of unity indicates mass is perfectly conserved during the time increment $\Delta t$.

Boundary fluxes in the FD models were evaluated with Darcy's Law at the interblock location between the boundary nodes $\left(B_{i}\right)$ and the first interior node $(i+1)$,

$$
F_{\alpha}^{\Delta t}=\sum_{B_{i}}\left[\lambda_{\alpha_{1-i} 2}\left(\frac{P_{\alpha_{1-1}}-P_{\alpha_{i}}}{\Delta z}\right) \Delta t\right]
$$

where interblock transmissibilities are evaluated as arithmetic means. Boundary fluxes in the FE model were calculated by back-substituting predicted pressures into the FE equations and solving for the boundary integral term. ${ }^{16}$

A cumulative mass balance error was also calculated using

$$
\text { \% cumulative error }(t)=100 \cdot 1 \cdot 0-\frac{M_{\alpha}^{t}-M_{\alpha}^{0} ;}{\sum_{\Delta t} F_{\alpha}^{\Delta t}}
$$

where $M_{\alpha}^{0}$ is the initial $\alpha$-fluid mass storage at time zero.

\section{Model verification}

Each of the three numerical models was verified against analytical solutions developed by McWhorter \& Sunada. ${ }^{21}$ These integral solutions describe the unsteady horizontal flow of two incompressible viscous fluids taking full account of capillary drive. Comparisons were made for one-dimensional unidirectional displacement of water by trichloroethane in soil system 1. The closed form solutions require a decaying organic influx given by:

$$
q_{\sigma}(0 . t)=A t^{-1 / 2}
$$

where $q_{o}$ is the organic injection rate, and $A$ is a constant associated with the steady boundary saturation $S_{g}=S_{n}(0, t)$. For all model comparisons $S_{g}$ was prescribed as 0.5 and the associated value of $A$ was 0.017187 .

Figure 2 compares the analytical and numerical solutions demonstrating close agreement for all three models. Small cumulative mass balance errors and deviations from the analytical solutions were observed in the pressure-based solutions. Thes errors can be attributed to the minimum $C_{\alpha}$ designation and are discussed in a later section. The above comparisons verify the ability of each of the three numerical codes to correctly solve the governing two-phase horizontal flow equations.
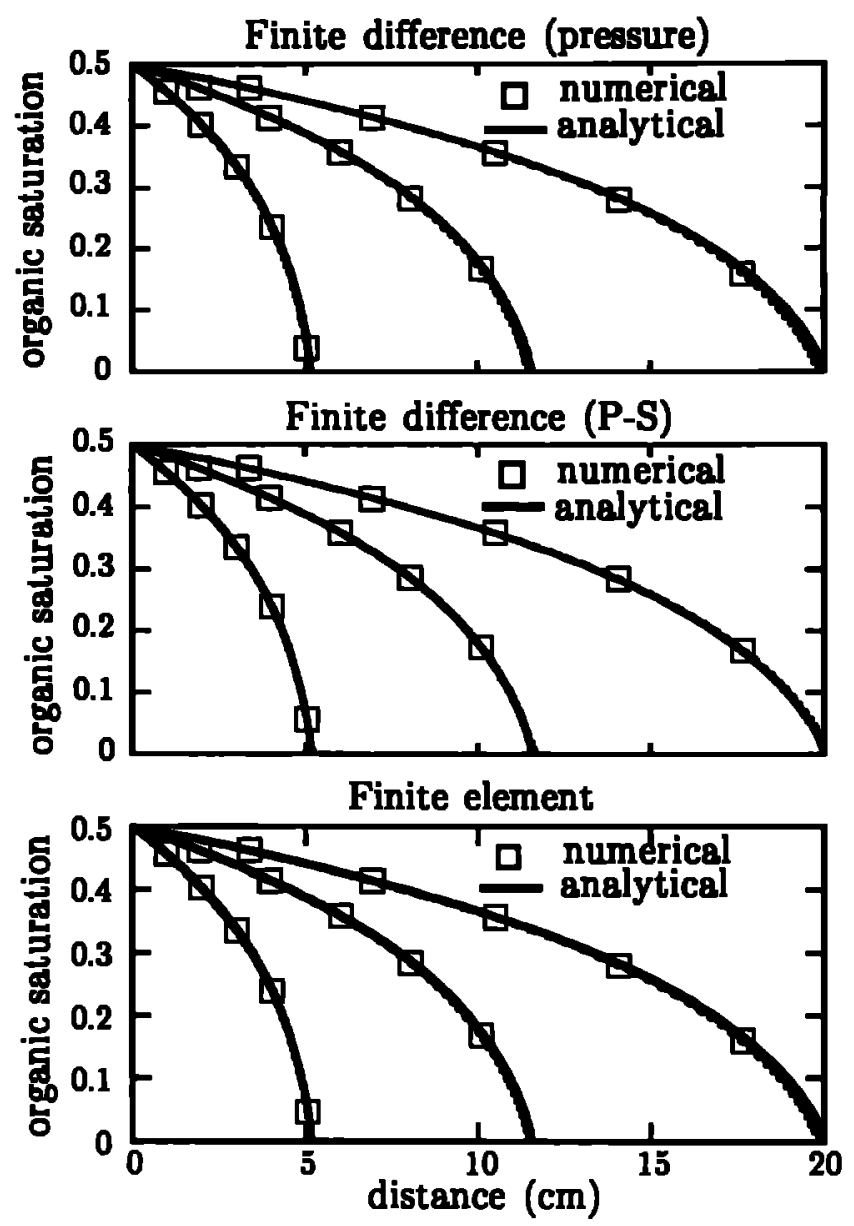

Fig. 2. Comparisons of analytical and numerical solutions for the 1D unidirectional water displacement problem at time 100 , 500 and $1500 \mathrm{~s}$.

\section{Influence of the initial conditions}

Implementation of the pressure-based formulation requires the specification of initial fluid pressures. When one of the fluids is absent from the domain, specification of the nonexistent fluid pressure is nonunique. For example, in contaminant hydrology problems a common initial condition is an uncontaminated aquifer devoid of NAPLs (i.e. $S_{o}=0$ ). In the case of two-phase flow any value of $P_{c} \leq 0$ is consistent with $S_{w}=1$. Consequently, there exists an ambiguity as to the correct specification of the initial nonexistent NAPL pressure distribution. Several approaches have been cited, while other researchers have neglected to fully disclose initial conditions. This issue has not been fully resolved within the literature.

One approach to the specification of $P_{\sigma}$ follows the classical method used in unsaturated flow modeling. Here the gas phase pressure is assumed to remain uniform at atmospheric conditions such that $P_{c}=-P_{x}$. By analogy, the absence of the organic liquid in the multiphase flow problem can be similarly characterized by setting $P_{g}=0$, again implying $P_{c}=-P_{w}$. Numerically, the negative capillary pressure is handled easily by 
Table 2. Initial and boundary conditions used in imulations

\begin{tabular}{|c|c|c|c|c|}
\hline Parameter & $\begin{array}{l}\text { Improper initial } \\
\text { conditions }\end{array}$ & $\begin{array}{c}\text { Organic } \\
\text { infiltration }\end{array}$ & $\begin{array}{l}\text { Restricted } \\
S_{o} \text { range }\end{array}$ & $\begin{array}{l}\text { Organic } \\
\text { drainage }\end{array}$ \\
\hline Column length $(\mathrm{cm})$ & 90 & 30 & 30 & 30 \\
\hline $\begin{array}{l}\text { Initial conditions } \\
\boldsymbol{P}_{\mathrm{k}}\end{array}$ & Hydrostatic & Hydrostatic & $\frac{\partial P_{w}}{\partial z}=\frac{\lambda_{o} \gamma_{c}-\lambda_{m} \gamma_{w}}{\lambda_{w}-\lambda_{w}}$ & $P_{o}-P_{c}$ \\
\hline $\begin{array}{c}P_{o} \\
P_{c} \\
\boldsymbol{S}_{w} \\
\text { Boundary conditions (top) }\end{array}$ & $\begin{array}{r}0.0 \\
-P_{w} \\
1.0\end{array}$ & $\begin{array}{l}P_{m} \\
0.0 \\
1.0\end{array}$ & $\begin{array}{c}P_{k}=P_{c} \\
P_{c}\left(S_{w}=0.9\right) \\
0.9\end{array}$ & $\begin{array}{l}\text { Hydrostatic } \\
16 \mathrm{~cm} \mathrm{H}_{2} \mathrm{O} \\
S_{w}\left(P_{c}\right)\end{array}$ \\
\hline $\begin{array}{l}P_{w} \\
P_{G} \\
S_{w} \\
\text { Boundary conditions (bottom) }\end{array}$ & $\begin{array}{c}0.0 \\
\gamma_{G} \cdot 15 \cdot 0 \mathrm{~cm} \\
S_{m}\left(P_{c}\right)\end{array}$ & $\begin{array}{c}0.0 \\
\gamma_{g} \cdot 15.0 \mathrm{~cm} \\
S_{w}\left(P_{c}\right)\end{array}$ & $\begin{array}{c}0.0 \\
\vartheta_{c} \cdot 15 \cdot 0 \mathrm{~cm} \\
S_{w}\left(P_{c}\right)\end{array}$ & $\begin{array}{c}\gamma_{\mathrm{m}} \cdot 15 \cdot 0 \mathrm{~cm} \\
P_{\mathrm{w}} \\
1 \cdot 0\end{array}$ \\
\hline $\begin{array}{l}\text { Boundary conditions (bottom) } \\
P_{w}\left(\text { dyne. } \mathrm{cm}^{2}\right)\end{array}$ & $\Upsilon_{\mathrm{m}} \cdot 90.0 \mathrm{~cm}$ & $\gamma_{\mathrm{m}} \cdot 30 \cdot 0 \mathrm{~cm}$ & $\frac{\lambda_{o} \tau_{o}-\lambda_{m} ?_{w}}{\lambda_{o}-\lambda_{w}} \cdot 30 \mathrm{~cm}$ & $P_{o}-P_{c}$ \\
\hline $\begin{array}{l}\left.P_{g} \text { (dyne } \mathrm{cm}^{2}\right) \\
S_{w}\end{array}$ & $\begin{array}{l}0.0 \\
1.0\end{array}$ & $\begin{array}{l}P_{w} \\
1 \cdot 0\end{array}$ & $P_{w w}-P_{c}$ & $\begin{array}{c}0.0 \\
S_{w}\left(P_{c}\right)\end{array}$ \\
\hline
\end{tabular}

setting $S_{n}=S_{w_{3 a r}}$. A uniform negative capillary pressure condition on the nonexistent organic fluid was employed in 2D simulations reported by Kuppusamy et al. $^{20}$

An alternative approach imposes the capillary pressure constraint in eqn (5); i.e. $P_{c}\left(S_{k}=1\right)=0$. Physically, the capillary pressure at $S_{w}=1$ must be zero since no interfacial curvature exists in the presence of only one fluid. This constraint, however, leads to a nonzero initial organic pressure distribution in the absence of the organic liquid. The $P_{c}=0$ approach has been used in both pressure-based and P-S formulations. 9.26

The influence of the initial organic pressure condition on mass balance accuracy was examined for a simulation of NAPL infiltration with the FD pressure-based code. A column was initially satuated with water in hydrostatic equilibrium and initial NAPL pressures were specified using either $\boldsymbol{P}_{o}=0$ or $\boldsymbol{P}_{c}=0$. Boundary and initial conditions are given in Table 2.

The progression of cumulative mass balance errors with time and the position of the saturation fronts is plotted in Fig. 3. When the initial NAPL pressure distribution is established from $P_{c}=0$, the cumulative mass balance error is small and diminishes over the course of the simulation. Small errors suggest the predicted saturation front is correctly positioned. In contrast, large mass balance errors are observed when a uniform initial pressure distribution is chosen. The cumulative errors grow over the course of the simulation as the NAPL advances deeper into the column and $P_{c}=-P_{w i}=\gamma_{u} \Delta h$ deviates further from the condition $P_{c}=0$. Propagation of the saturation front is significantly impeded in this case. Thus it can be concluded that the correct initial pressure specification for an absent organic phase is $\boldsymbol{P}_{o}=\boldsymbol{P}_{n}$.

When both fluids are initially present, the initial pressure field can be established from any initial saturation distribution by solving the sum of the steady state flow equations: $:^{24}$

$$
\begin{array}{r}
\nabla \cdot\left(\rho_{a} \lambda_{o}+\rho_{w} \lambda_{w}\right) \nabla P_{w}+\rho_{o} \lambda_{\sigma} \nabla P_{\sigma} \\
-\left(\rho_{c} \lambda_{o} \gamma_{o}-\rho_{w} \lambda_{w} \gamma_{w}\right) \nabla z_{-}^{-}=0
\end{array}
$$

\section{Influence of the capacity coefficient}

The influence of $C_{\alpha}$ on mass balance accuracy was examined in simulations of NAPL infiltration using the boundary and initial conditions in Table 2 . The resulting cumulative mass balance errors are plotted in Fig. 4 as a
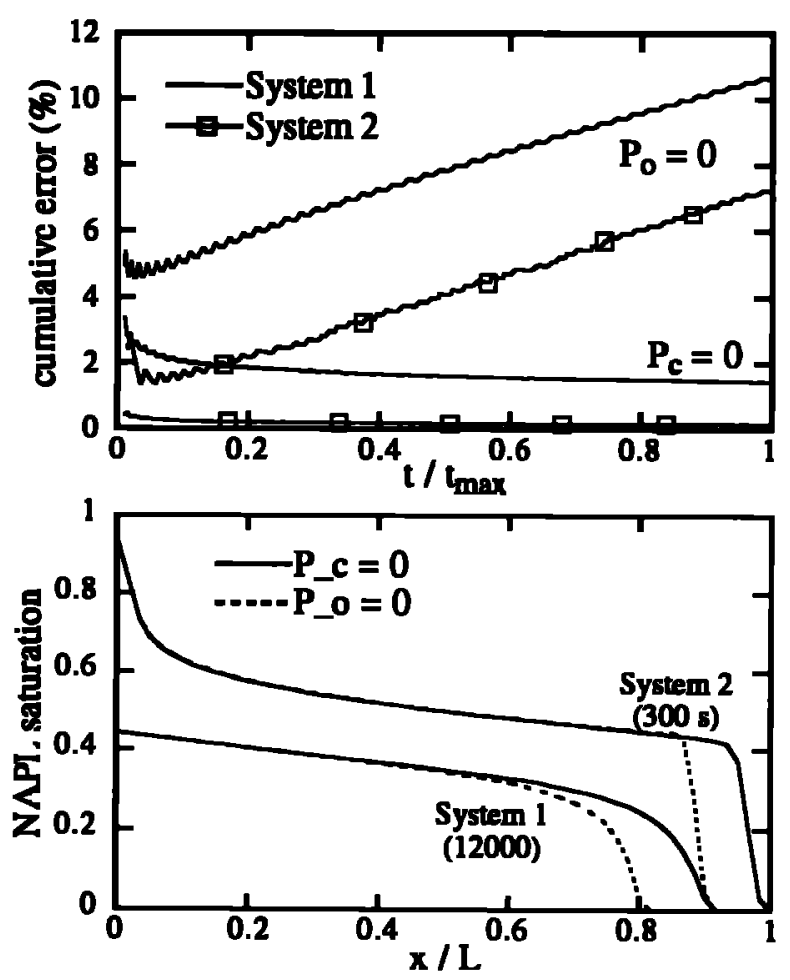

Fig. 3. Effect of initial NAPL pressure specification on cumulative mass baolance error and position of the saturation front. $\Delta z=60 / L$ and $\Delta l_{\max }=20.0$ and $0.5 \mathrm{~s}$ for systems 1 and 2. respectively. 

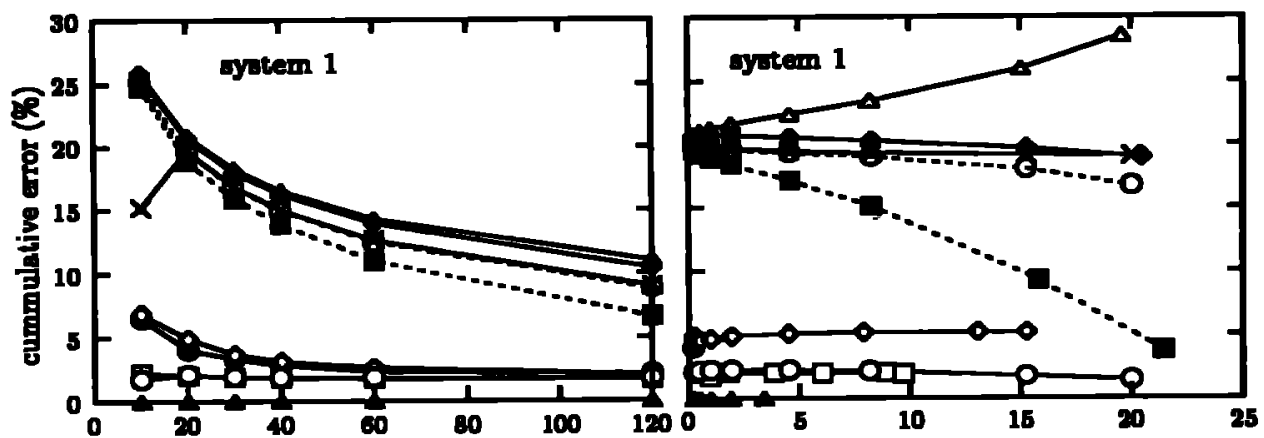

- O-FD - presure

$\sim$-FD - PS

A-enalytical $(w=1.0)$

- W-time averege $\mathrm{Pc}(w=0.5)$

-- previoun time Pe $(w=0.0)$

--o-mean Pc
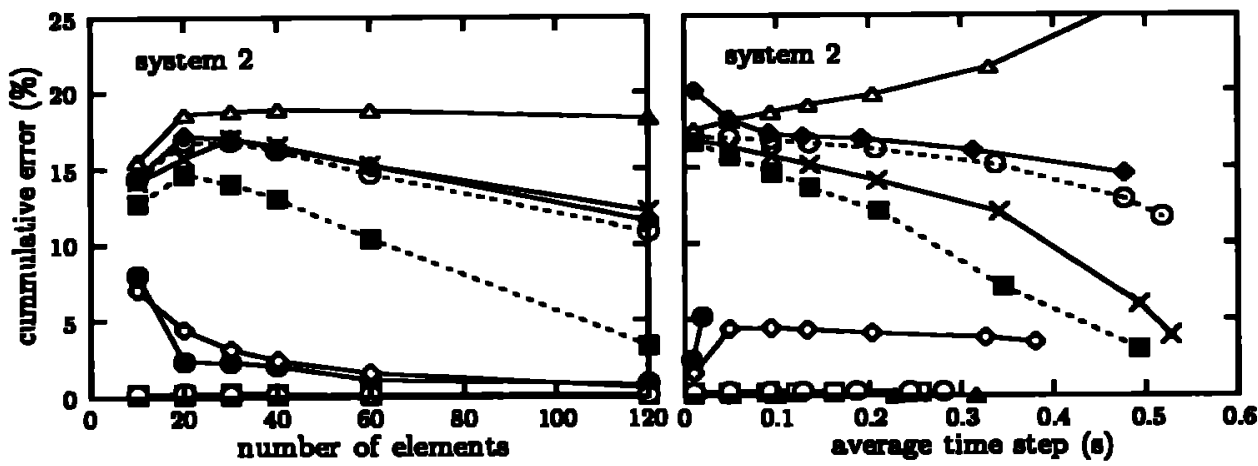

-traditional chord slope

- -distributed chord alope

-row diotributed chord alopo

-OFE chord alope

Fig. 4. Effect of $\Delta z$ and $\Delta t$ on cumulative mass balance error at time 900 and $30 \mathrm{~s}$ in systems 1 and $2 . \Delta t_{\text {rax }}$ in element size comparisons are 1.0 and $0.1 \mathrm{~s}$, respectively. Time step comparisons use a 20 -element grid.

function of $\Delta t$ and $\Delta z$. Both FD schemes and those FE schemes which preserve expansion of the saturation derivative demonstrate good mass balance accuracy, with errors on the order of $5 \%$ or less. The mass balance error does not appear to be adversely sensitive to $\Delta t$ or $\Delta z$ over the range examined. In contrast, FE schemes using the scs or analytical approximations produce mass balance errors on the order of $20 \%$ and exhibit greater sensitivity to $\Delta t$ and $\Delta z$. The mass balance errors in these schemes can be attributed primarily to the temporal discretization terms because spatial discretization is identical for all models and mass balance accuracy in the conservative schemes is relatively insensitive to $\Delta z$.

Figure 5 compares the predicted saturation distributions of representative schemes with the P-S solutions. Recall that the P-S model does not require evaluation of a capacity coefficient in this two-phase case and hence exhibited excellent mass balance accuracy. Predictions from pressure-based formulations agree closely with the

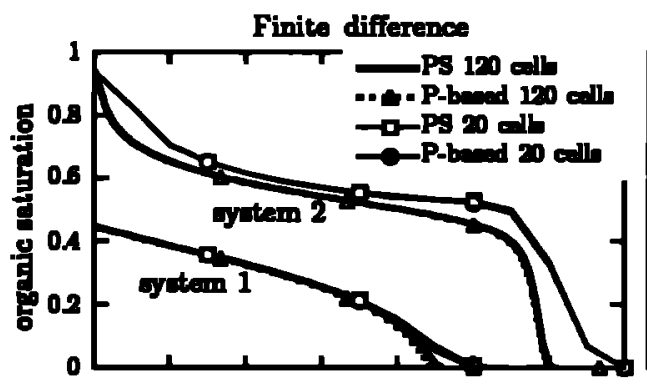

FE - anelytical $(w=0.0)$

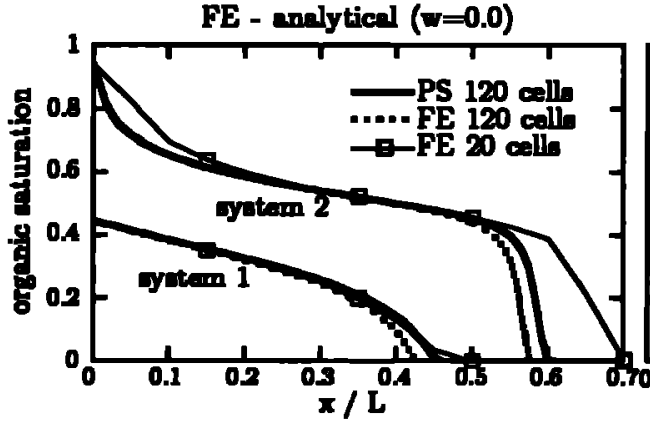

FE - triditional chord alope

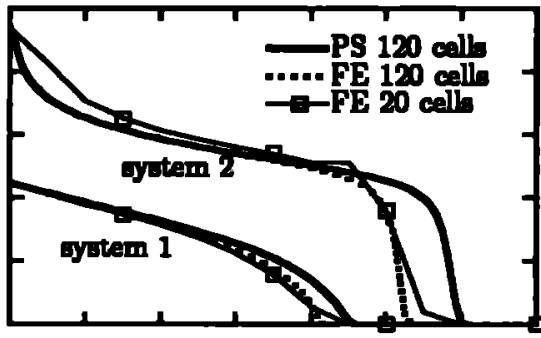

FE - row diotributed chord lope

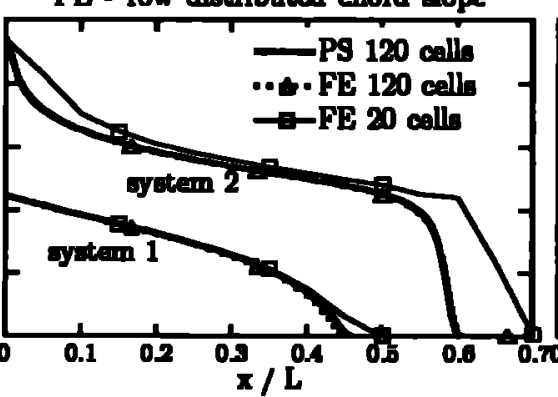

FE - analytical ( $w=1.0)$

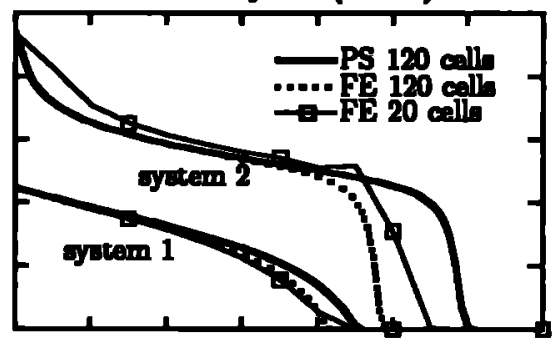

FE - finite element chord alope

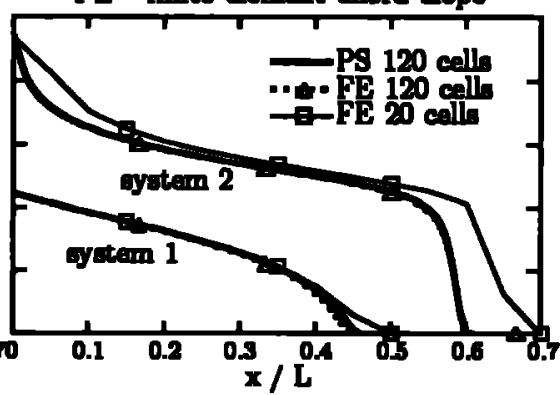

Fig. 5. Predicted XAPL saturation distribution with selected capacity coefficient approximation forms. Tume $=900$ and $30 \mathrm{~s}$ in systems 1 and 2. 
Table 3. Cumulative mass balance errors in sumulation of NAPL infiltration over the generally linenr reglon of $\boldsymbol{P}_{c}\left(\boldsymbol{S}_{\mathbf{0}}<0.0\right)$

\begin{tabular}{lll}
\hline \multicolumn{1}{c}{ Algorithm } & \multicolumn{2}{c}{ Cumulative error (\%) } \\
\cline { 2 - 3 } & \multicolumn{1}{c}{ System 1 } & System 2 \\
\hline FD, pressure based & 0.1 & $8.6 \times 10^{-3}$ \\
FD, P-S formulation & $5.0 \times 10^{-3}$ & $3.1 \times 10^{-3}$ \\
FE, analytical $(w=1 \cdot 0)$ & 0.09 & 0.86 \\
FE, time average $\boldsymbol{P}_{c}\left(\boldsymbol{w}^{\prime}=0.5\right)$ & 0.16 & 1.47 \\
FE, previous time $\boldsymbol{P}_{c}\left(w^{\prime}=0.0\right)$ & 0.33 & 2.09 \\
Fe, mean $\boldsymbol{P}_{c}$ & 0.15 & 1.46 \\
Fe, scs & 0.08 & 1.26 \\
Fe, dcs & 0.26 & 0.96 \\
Fe, rdcs & 0.16 & 0.77 \\
Fe, fecs & 0.32 & 0.01 \\
\hline
\end{tabular}

Time $=900$ and $30 \mathrm{~s}$ for systems 1 and 2 , respectively.

P-S solutions for the FD model and for those FE schemes which preserve elemental expansion of the saturation derivative, i.e. precisely those schemes which yield good mass balance accuracy. The non-conservative scs and analytical FE schemes under-predict the position of the saturation fronts in comparison with the mass conservative schemes.

Errors observed in the presure-based FE solutions can be explained by failure to preserve expansion of the discretized saturation derivative. This emror can be quantified with an elemental residual, $\overbrace{\epsilon_{\alpha}}$, between the saturation derivative and the expanded form in eqn (27),

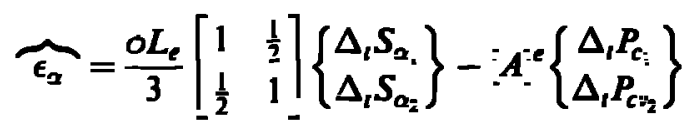

The element-dependent $C_{\alpha}^{d k s}, C_{\alpha}^{\text {rdcs }}$, and $C_{\alpha}^{\text {FEcs }}$ approximations were developed on the basis of epsilon $n_{\alpha}=0$, and, hence, demonstrate good mass balance accuracy in Figs 4 and 5. The scs and analytical approximations do not necessarily satisfy expansion of the saturation derivative, in which case $\overbrace{\epsilon_{\alpha}}$ is nonzero. These latter approximations are continuous at the elemental boundaries such that $\overbrace{\epsilon_{\alpha}}$ may be assembled and examined on a nodal basis:

$$
\begin{aligned}
& \epsilon_{\alpha_{.}}=\frac{o L_{e}}{6}\left(\Delta_{t} S_{\alpha_{.-1}}-4 \Delta_{t} S_{\Omega}-\Delta_{t} S_{\alpha_{--1}}\right)
\end{aligned}
$$

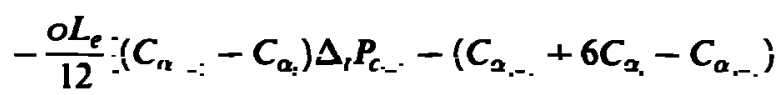

$$
\begin{aligned}
& \times \Delta_{l} P_{c .}-\left(C_{\mathrm{n}_{i}}-C_{\mathrm{n}_{.-}}\right) \Delta_{l} P_{c_{i--:}-}
\end{aligned}
$$

Making the substitution $\Delta_{l} P_{c_{\mathrm{f}}}=\Delta_{l} S_{\alpha} / C_{\alpha,}^{-}$, eqn (45) may be rearranged as

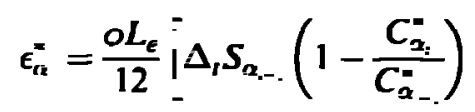

$$
\begin{aligned}
& -\Delta_{t} S_{\alpha_{1}}\left(2-\frac{C_{\alpha_{1-i}}^{-}}{C_{\alpha_{s}}^{-}}-\frac{C_{\alpha_{t-i}}^{-}}{C_{\alpha_{s}}^{-}}\right) \\
& \left.+\Delta_{t} S_{\alpha_{t-}}\left(1-\frac{C_{\alpha_{i}}^{*}}{C_{\alpha_{i-:}}^{*}}\right)\right]
\end{aligned}
$$

where $C_{\alpha_{i}}^{-}$indicates approximations by the analytical or scs methods. When $\epsilon_{\alpha_{i}}^{-}$is zero, the expansion in eqn (22) is exact at node $i$ and $\mathrm{MBE}=1$. A positive value of $\epsilon_{\alpha_{i}}$ implies $\partial S / \partial t>C_{\alpha} \partial P_{c} / \partial t$; the predicted change in saturation is less than the true (mass conservative) change, or $\mathrm{MBE}<1$. Conversely, $\epsilon_{\alpha_{1}}^{*}<0$ signifies an over-prediction in saturation change and $\mathrm{MBE}>1$.

Examination of eqn (46) indicates $\epsilon_{\alpha_{i}}^{=}$depends on the degree of nonlinearity in $P_{c}\left(S_{i x}\right)$; similar findings were presented by Milly. ${ }^{22}$ Nonlinearity in the van Genuchten form of $P_{c}\left(S_{w}\right)$ occurs in two regions (see Fig. 1): at the saturation front during the primary displacement of the wetting phase by a nonwetting NAPL, and near $S_{w}=S_{r n}$. For the simulated organic infiltration problems, the controlling nonlinearity appears to be the former region; the discrepancy in predicted NAPL saturation distributions occurs primarily in the positioning of the saturation front and the water saturation behind the front is well above $S_{r n}$. At the saturation front, $\left|C_{\alpha_{1-}} \geq C_{\alpha_{i}}\right| \geq \mid C_{\alpha_{-}-:}$for flow in the direction of $i-1$ to $i-1$, resulting in a nonzero $\epsilon_{\boldsymbol{s}_{i}}^{-}$. The inexact expansion of $\partial S / \partial t$ over the nonlinear region of $P_{c}\left(S_{w}\right)$ explains the poor mass balance accuracy evidenced in Figs 4 and 5.

If $P_{c}\left(S_{w^{\prime}}\right)$ is linear (i.e. $\left.C_{\Omega_{i--}}^{-}=C_{\underline{s}_{i}}^{-}=C_{\Omega_{i-1}}^{-}\right)$, the residual $\epsilon_{\alpha,}^{*}$ is zero and any of the $C_{\alpha}^{-}$forms should yield good mass balance accuracy. This hypothesis was tested by increasing the initial uniform organic saturation from zero to 0.1 , forcing organic infiltration over the generally linear segment of $P_{c}\left(S_{w}\right)$ in Fig 1. Lsing initial and boundary conditions given in Table 2 , predictions were obtained on a 60-element grid with maximum time steps of 1.0 and $0.1 \mathrm{~s}$ for systems 1 and 2. The resulting mass balance errors are listed in Table 3 and demonstrate that good accuracy is obtained with all $C_{\Omega}$ schemes when $P_{g}\left(S_{k}\right)$ is linear. The predicted saturation distributions agreed closely for all cases.

The influence of nonlinearity in $P_{c}\left(S_{n}\right)$ on mass balance accuracy was investigated by plotting incremental MBE against time in Fig 6. The P-S formulation exhibits constant $M B E=1$ and excellent cumulative mass balance accuracy. In comparison, all pressurebased solutions display oscillatory behavior in $\mathrm{MBE}^{\prime}(t)$ with varying frequency and amplitude. The $d c s$ and $r d c s$ forms exhibited especially large amplitudes in $\operatorname{MBE}(t)$ which could be damped by increasing the convergence criterion. In the other schemes the convergence criterion had little influence on the amplitude of MBE. For all schemes. grid size had the greatest effect on frequency and amplitude of oscillations. Time step size had little 

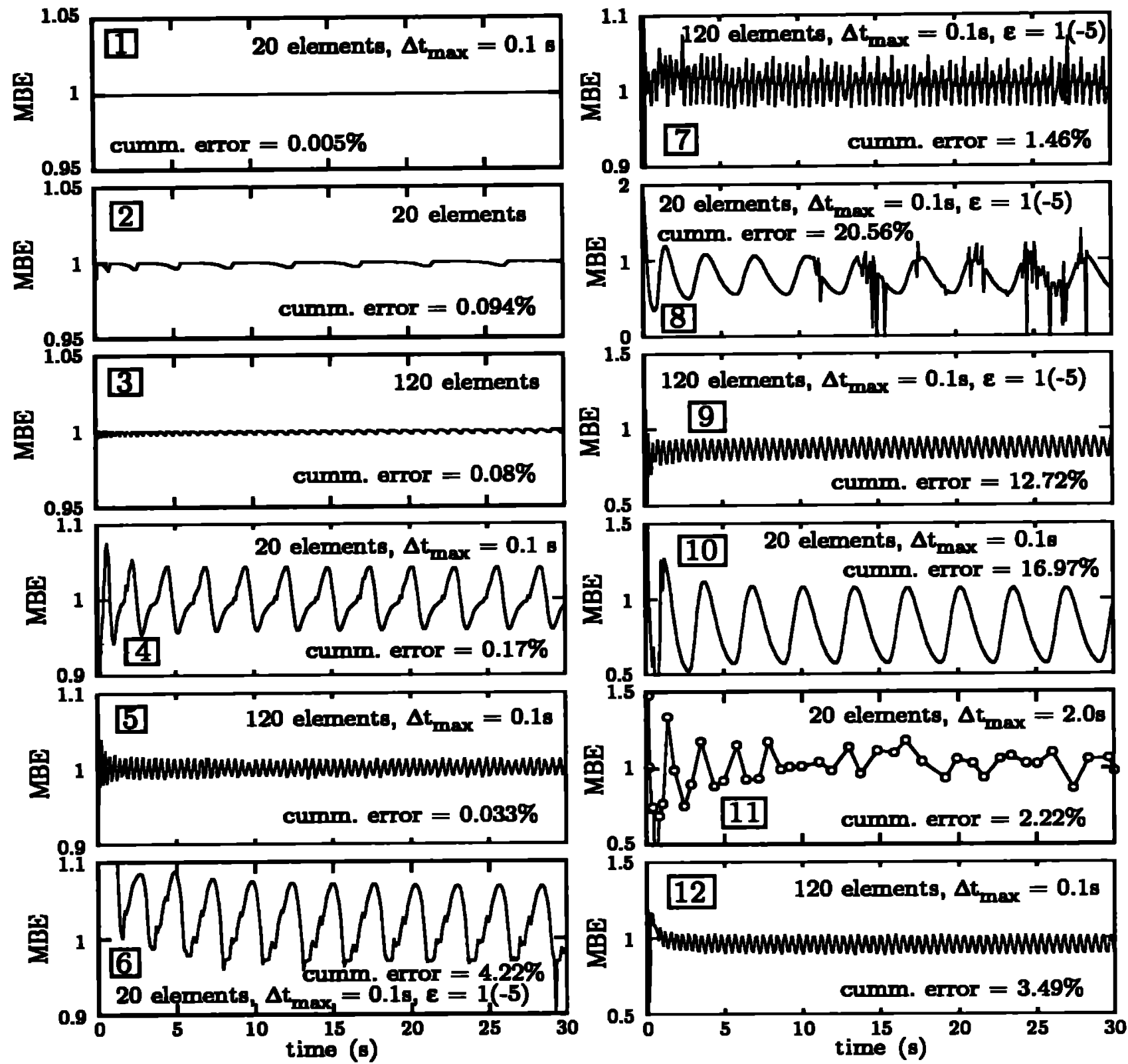

Fig. 6. Mass balance errors vs time from simulations with system 2 data: 1, P-S formulation; 2-3, FD pressure formulation; 4-5, FEcs formulation; 6-7, FErdcs scheme; 8-9, FE pressure based model with scs approximations; 10-12, FE pressure based model with analytical $C_{\alpha}\left(w^{\prime}=0\right)$ approximations.

overall influence in the mass conservative cases, but greatly influenced cumulative mass balance accuracy in models employing the analytical capacity forms.

Heuristic explanations can be offered for the observed behavior in $\operatorname{MBE}(t)$ by examining the nodal residual given by eqn (46). Consider the analytical and scs approximations. Figure 7 illustrates the capacity coefficients of these schemes at three nodes at the toe of the saturation front. When the saturation front initially enters a new element $i-1$ at time $t-1$, both $S_{0}$, , and $C_{1-1 . i-i}^{*}$ are small, whereas immediately behind the front $S_{s_{: t-:}}$ and $I C_{i, t-1}^{-}$are relatively large. Hence at the saturation front $C_{t . t-1}^{*} 1 / C_{l-1 . t-:}^{*} \gg 1$ and behind the front $C_{l, t-1}^{*} / C_{l-: . t-1 .}^{*} \approx 1$. The nodal residual $\epsilon_{i}^{*}$ is thus nonzero yielding $\mathrm{MBE} \neq 1$.
The relative magnitude of the last two terms in eqn (46) alternate in response to the nodal saturation values. As the front initially enters node $i-1$ there is a period of pressure build-up without appreciable desaturation. i.e. $\Delta_{t} S_{i-i} \approx 0$. The second term in eqn (46) increases in relative magnitude producing a positive slope in the $\operatorname{MBE}\left(t_{j}\right)$. Once the entry pressure is exceeded, $\Delta_{l} S_{i-\text { : }}$ increases, correspondingly increasing the relative magnitude of the last term in eqn (46). This produces a negative slope in $\operatorname{MBE}_{(}(t)$. The interplay between the last two terms in eqn (46) as the saturation front enters and fills each element accounts for the oscillatory behavior of $\operatorname{MBE}(t)$. Reducing the element size increases the number of elements encountered by the saturation front, increasing the frequency of the $\operatorname{MBE}^{\prime} t ;$. A smaller 

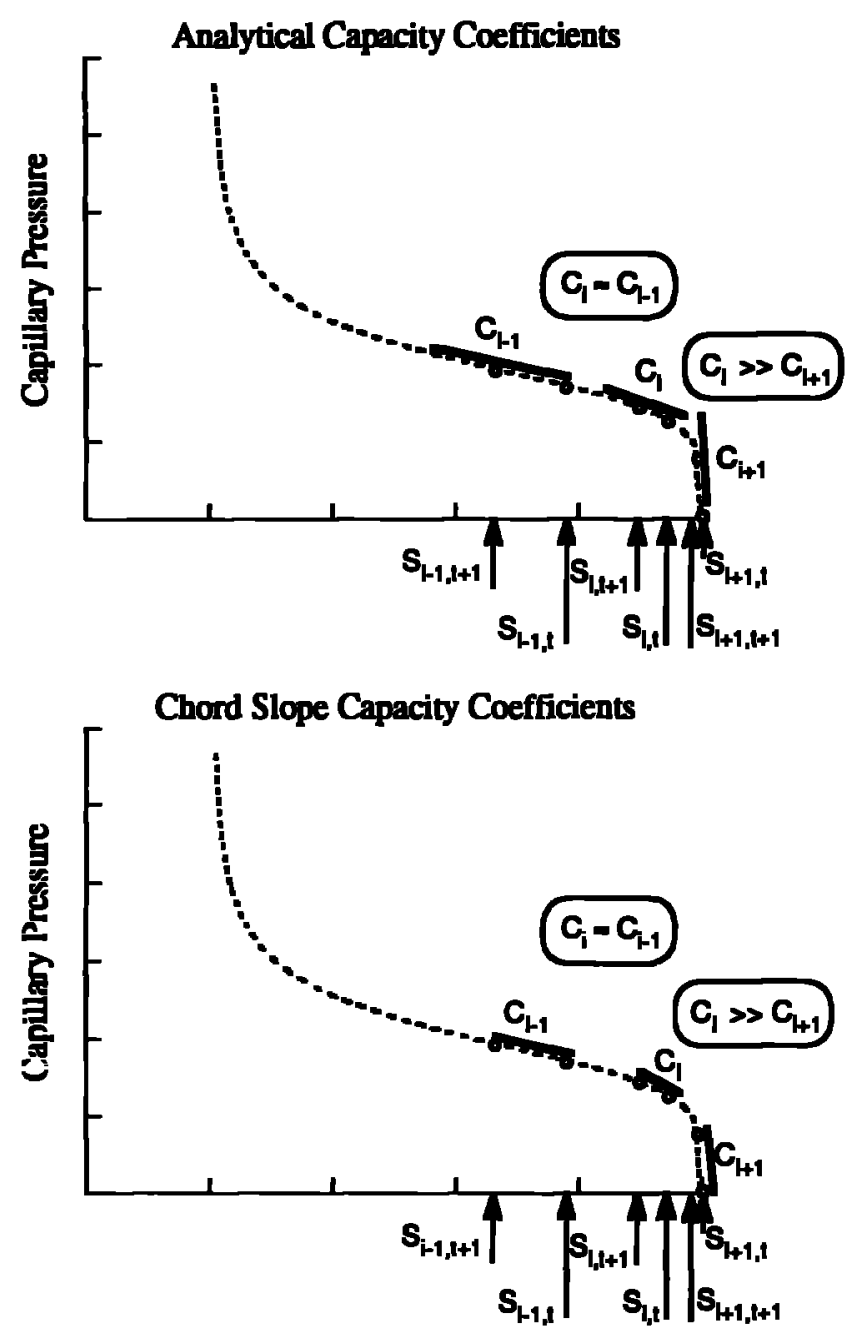

Fig. 7. Analytical and scs $C_{\alpha}$ approximations.

element size also requires less time to establish the entry pressure, reducing the amplitude of $\operatorname{MBE}(l)$.

The FEcs, dcs and rdcs approximations cannot be depicted graphically and thus the discussion above is not necessarily applicable to these schemes. Similar oscillatory behavior in Fig 6, together with an observed damping when $C_{a}$ is constant, suggests that these fluctuations may likewise be attributable to the nonlinearity in $\boldsymbol{P}_{e}\left(\boldsymbol{S}_{\mathrm{w}}\right)$.

The FD pressure-based model produced a saw tooth wave pattern in $\operatorname{MBE}(t)$ rather than the sinusoidal waves observed with the FE models. This result can be explained by considering the nodal residual obtained by rearranging eqn (23):

$$
\epsilon_{\boldsymbol{s}_{i}}^{\mathrm{FD}}=\Delta_{l} S_{\alpha_{i}}-C_{\alpha_{i:-1}}^{\mathrm{sc}} \Delta_{l} P_{c_{i}}
$$

The scs approximation is developed by imposing $\epsilon_{x_{i}}^{\mathrm{FD}}=0$. If $C_{\alpha_{:-1}}^{\mathrm{scs}}$ is evaluated in a fully implicit manner, no oscillatory behavior is expected, i.e. $\epsilon_{\boldsymbol{\alpha}_{1}}^{\mathrm{FD}}=0$ and $\mathrm{MBE}(t)=1$. In the numerical scheme, however, evaluation of $C_{\mathrm{\alpha}_{i:-1} \text {, }}^{\text {ses }}$ is lagged by one iteration. This leads to an under-evaluation of $C_{\alpha_{:-1}}^{s c s}$ at the saturation front and results in non-

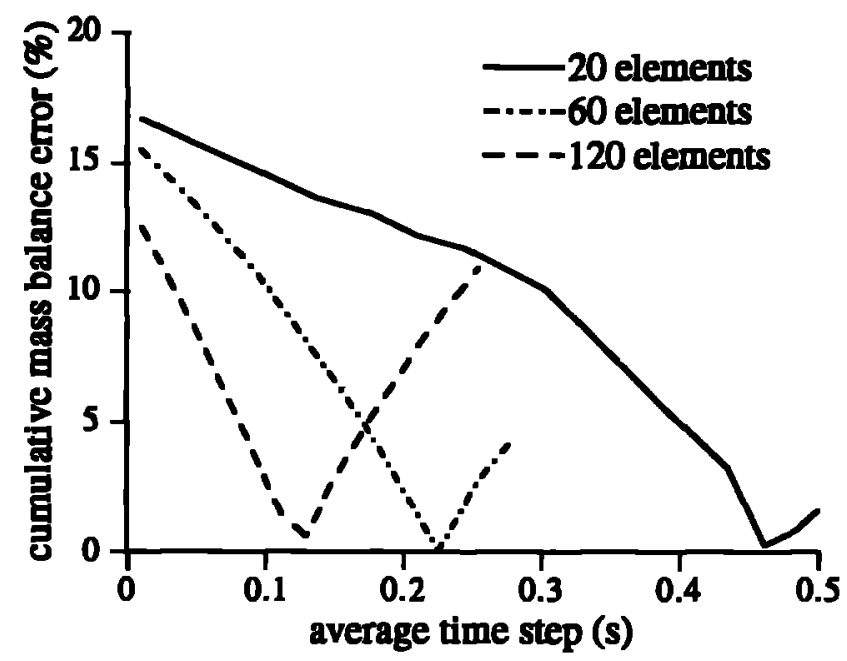

Fig. 8. Cumulative mass balance error at $t=30 \mathrm{~s}$ versus average time step size. FE simulations are with System 2 data using analytical $(w=0)$ capacity coefficients.

negative $\epsilon_{\alpha_{1}}^{\mathrm{FD}}$. Consequently, $\mathrm{MBE}(t)$ is bounded by $M B E \leq 1$, producing the saw tooth wave pattern.

Figure 6 reveals that oscillations in $\operatorname{MBE}(t)$ do not necessarily result in unacceptable cumulative mass balance accuracy. For the mass conservative FE schemes, $\operatorname{MBE}(t)$ is generally centered on $M B E=1$; it is displaced from $M B E=1$ in the non-conservative schemes. The magnitude of the displacement correlates with the cumulative error and is influenced by both $\Delta z$ and $\Delta t$. This behavior is also exhibited in Fig 4 which shows cumulative errors in the non-conservative schemes decrease with decreasing $\Delta z$ or increasing $\Delta t$. The influence of $\Delta t$ is surprising because the improved accuracy occurs despite the expected increase in truncation error which is dominated by terms like $\left(-\Delta t / 2+\Delta t^{2} / 3\right) S_{2}^{\prime}{ }^{6}$. Moreover, the magnitude of accuracy improvement appears to be proportional to the explicit weighting on the previous time step information; $\boldsymbol{w}=\mathbf{0}$ produces the greatest improvement and $w=1$ exhibits no improvement.

Additional analysis was undertaken to explore cumulative mass balance errors from the FE analytical $C_{\alpha}$ scheme $(w=0)$ for a range of element and step sizes. Figure 8 reveals that cumulative accuracy consistently improves with increasing $\Delta t$ until the error is virtually eliminated at some critical average $\Delta t$. At large time steps, however, the trend reverses yielding increasing error with increasing $\Delta t$.

The unanticipated influence of time step can be explained by again considering the last two terms in the nodal residual eqn (46). If the previous time step solutions are employed to evaluate the analytical capacity terms, these terms are constant, independent of $t-1$ nodal saturations. Moreover, the ratio $C_{i, t-1}^{*} / C_{l-1 . t-1}^{*}$ is large because ' $C_{i-1 . t}$ is undefined and thus assigned a small nonzero value. Now examine the $\Delta_{t} S_{i-1}$ and $\Delta_{t} S_{i}$ terms. For small $\Delta t$, the time step is 


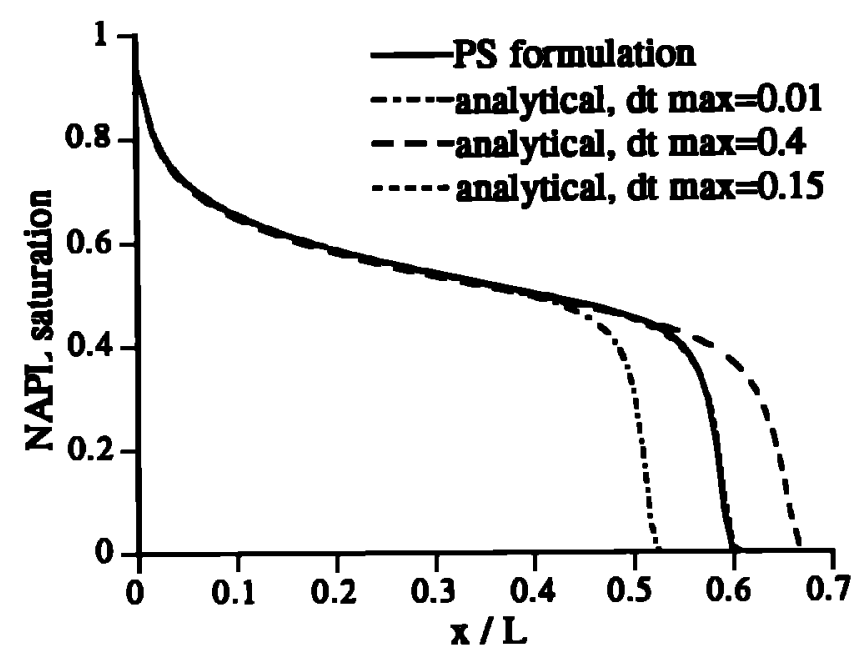

Fig. 9. Effect of maximum time step size on predicted NAPL saturation distributions with the FE scheme employing analytical $(w=0)$ capacity coefficients. Simulations employ System 2 data and a 120-element grid.

insufficient for the pressure build-up at node $i-1$ to exceed the entry pressure. Consequently $\Delta_{l} S_{i-1} \approx 0$, the second term in eqn (46) is dominant, and $\epsilon_{i}^{*}$ is positive. This analysis is supported by Figs 6-10 which shows $\operatorname{MBE}(t)$ lies below $\mathrm{MBE}=1$. For large $\Delta t$ the entry pressure is exceeded within a single time step and $\Delta_{l} S_{i-1}$ is nonzero. Thus the last term in eqn (46) becomes dominant, $\epsilon_{i}^{-}$is negative, and $\operatorname{MBE}(t)$ is offset above $\mathrm{MBE}=1$ in Figs 6-11. At some critical $\Delta t$ there is a cancelling of the last two terms in eqn (46) yielding $\epsilon_{i}^{-}=0$ and good cumulative mass balance accuracy.

The magnitude and average sign of $\epsilon_{i}^{-}$is reflected in the positioning of the saturation front. Figure 9 shows that the choice of $\Delta t$ can impede, advance, or match the correct position of the saturation front. The dependence of cumulative error on time step size may partially explain the improved mass balance accuracy obtained with the modified chord slope approximation of Kaluarachchi \& Parker $^{17}$ which effectively increases the explicit weighting.

\section{Infiuence of the minimum capacity coeficient}

A common problem in contaminant hydrology is the infiltration of a NAPL into an uncontaminated aquifer $\left(S_{o}=0\right)$. In the absence of the NAPL, $P_{c}=0$ and $C_{\alpha}=0$. Because the pressure-based formulation tends toward singularity as $\boldsymbol{P}_{c}$ approaches zero, a small 'dummy' $C_{\alpha}$ or $S_{o}$ is typically specified. ${ }^{1.6 .17}$ Kueper \& Frind $^{19}$ argue that a major advantage of the P-S formulation is that it can easily accommodate conditions of a nonexistent NAPL without fictitious representation of the state variables.

The influence of the minimum $C_{\alpha}$ on mass balance accuracy was studied in simulations of NAPL infiltration in a 40-element domain, using initial and boundary conditions given in Table 2. Figure 10 shows the variation of cumulative mass balance error with the $\log \left(\min C_{\alpha}\right)$. A measure of the relative computational effort $(\log$ [number of time steps $x$ the total number of iterations]) ${ }^{22}$ is also plotted.

Excellent mass balance accuracy was obtained with each of the mass conservative $C_{\alpha}$ forms when min $C_{a} \leq 1(-6)$; mass balance accuracy degenerates dramatically when $\min C_{\alpha}>1(-6)$. Thus, the fictitious or 'dummy' $C_{\alpha}$ can be made sufficiently small such that

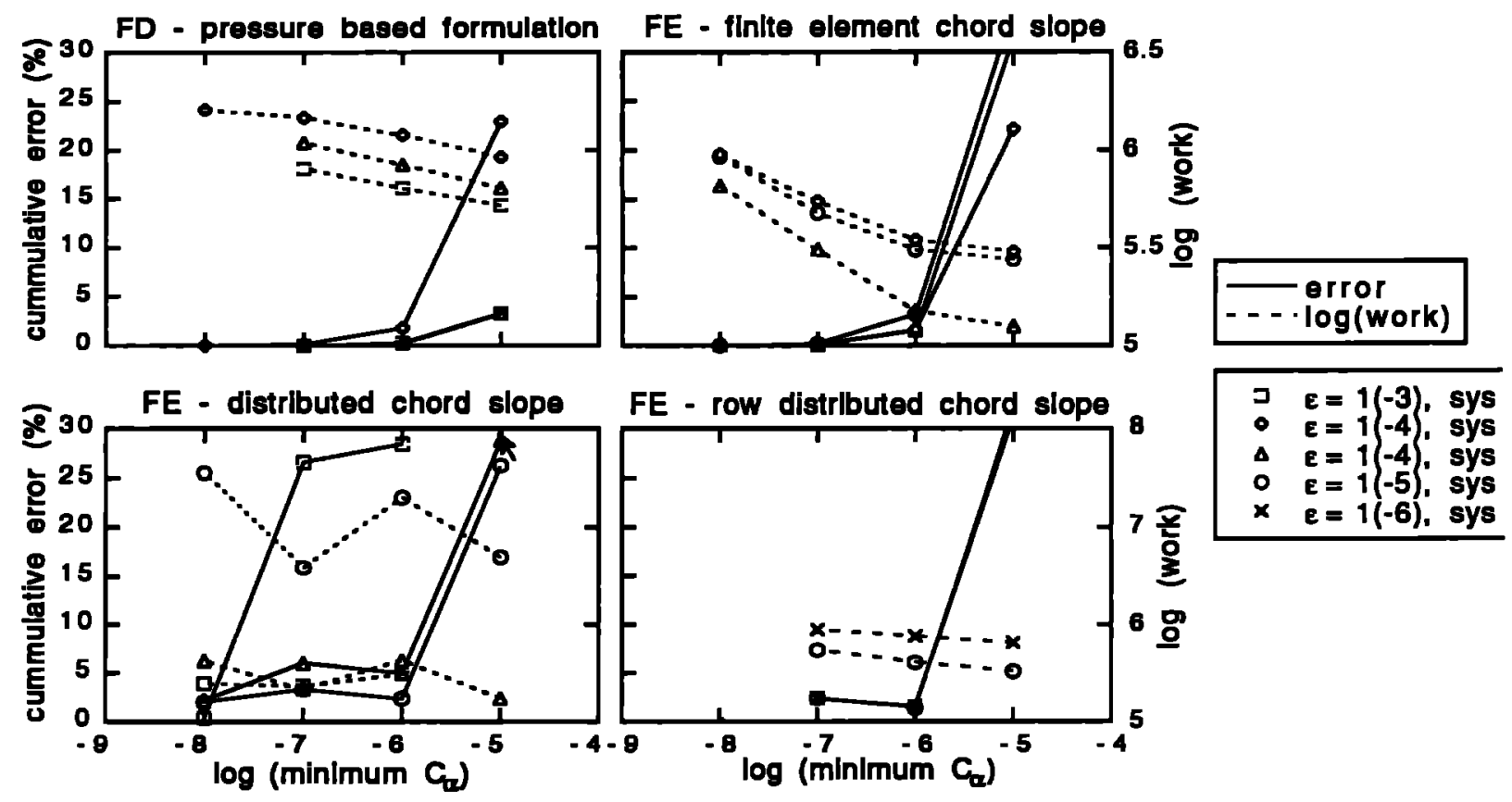

Fig. 10. Effect of min $C_{\alpha}$ on cumulative mass balance accuracy (left ordinate) and relative computational work (right ordinate). Simulations are with System 2 data. 
Table 4. comparison of cumnlative mass balance errors and computhtional work from consistent and mass humped formulations in the NAPL infiltration problem whin System 2 datn

\begin{tabular}{|c|c|c|c|c|}
\hline \multirow[t]{2}{*}{$C_{\alpha}$ form } & \multicolumn{2}{|c|}{ Consistent form } & \multicolumn{2}{|c|}{ Mass lumped form } \\
\hline & Cumulative error (\%) & Worke & Cumulative error (\%) & Work $^{\circ}$ \\
\hline $\begin{array}{l}\text { FE chord slope } \epsilon=1(-4)^{-} \\
\min C_{\alpha}=1(-5) \\
\min C_{\alpha}=1(-6) \\
\min C_{\alpha}=1(-7)\end{array}$ & $\begin{array}{l}1 \cdot 58 \\
0 \cdot 11 \\
0 \cdot 10\end{array}$ & $\begin{array}{l}5.18 \\
5.49 \\
5.82\end{array}$ & $\begin{array}{l}3.36 \\
0.21 \\
0.03\end{array}$ & $\begin{array}{l}5.46 \\
5.55 \\
5.68\end{array}$ \\
\hline $\begin{aligned} \text { FEdcs } \epsilon^{-} & =1(-4)^{\prime} \\
\min C_{\alpha} & =1(-5) \\
\min C_{\alpha} & =1(-6) \\
\min C_{\alpha} & =1(-7) \\
\min C_{\alpha} & =1(-8)\end{aligned}$ & $\begin{array}{r}4.99 \\
5.99 \\
2.13\end{array}$ & $\begin{array}{l}5.63 \\
5.33 \\
5.62\end{array}$ & $\begin{array}{l}1.09 \\
0.85 \\
1.00 \\
1.03\end{array}$ & $\begin{array}{l}5.52 \\
5.62 \\
5.72 \\
5.92\end{array}$ \\
\hline $\begin{aligned} \text { FErdcs } \bar{\epsilon} & =1(-4) \\
\min \bar{C}_{\alpha} & =1(-5) \\
\min C_{\alpha} & =1(-6) \\
\min C_{\alpha} & =1(-7) \\
\min C_{\alpha} & =1(-8)\end{aligned}$ & $\begin{array}{l}b \\
b \\
b \\
b\end{array}$ & & $\begin{array}{l}4-56 \\
0.46 \\
0.96 \\
1.03\end{array}$ & $\begin{array}{l}5.52 \\
5.58 \\
5.77 \\
5.91\end{array}$ \\
\hline
\end{tabular}

Work $=\log$ (number of iteration $x$ number of time steps).

${ }^{b}$ Would not converge.

solution accuracy is not adversely affected. Although soil type did not appreciably affect these observations for the examined simulations, it is possible that the optimal selection of $\min C_{\alpha}$ is problem-specific. Consequently the specification of $\min C_{\alpha}$ should be considered a possible source of mass balance error and examined with appropriate sensitivity studies.

The plots in Fig 10 also provide evidence that the FD and FEcs methods are more stable and accurate than the FE $d c s$ and $r d c s$ schemes. The FD and FEcs methods produce excellent mass balance results with a welldefined trade-off between mass balance accuracy and computational work. In comparison the $d c s$ and $r d c s$ methods produce somewhat poorer mass balance accuracy, with errors generally ranging above $1 \%$. With these schemes, computational work appears to be less dependent on $\min C_{\alpha}$. In the case of the rdcs method, the range in which accurate converged solutions could be obtained is relatively narrow.

\section{Influence of mass lumping}

Mass lumping procedures have been advocated for FE unsaturated flow models to control oscillatory solutions in advection dominated problems. ${ }^{722}$ The diagonalization of the mass matrix introduces additional dissipation in the stiffness matrix which damps oscillatory solutions. ${ }^{30}$ In general, mass lumping tends to improve solution stability at the expense of some loss in accuracy. ${ }^{16.30}$

Mass lumping procedures were implemented in connection with the FEcs, dcs, and rdcs schemes simulating organic infiltration in system 2 data on a 40-element grid. Table 4 lists the resulting cumulative mass balance errors from the lumped and consistent formulations. Mass balance errors in the lumped models agree favorably with those obtained from the consistent models, and the predicted saturation distributions from all models are in close agreement.

Mass lumping was found to improve stability of the numerical solutions. The des and rdcs methods benefitted greatly from mass lumping, exhibiting an oscillation free incremental $\operatorname{MBE}(t)$ which was close to unity after an initial adjustment period. The improved stability of the lumped schemes expands the convergence range over $\epsilon$ and $\min C_{\alpha}$, especially with the rdcs approach where none of the consistent forms would converge for $\epsilon \leq 1(-4)$. Greater stability is also accompanied by improved cumulative mass balance accuracy and generally increased computational work.

The mass lumped FCcs method is equivalent to the implicit FD approach and hence produces a saw tooth wave in $\operatorname{MBE}(t)$ similar to that observed in the FD solutions. Mass lumping in this scheme results in some loss of cumulative mass balance accuracy and generally increased computational effort. A possible source of the error increase is the $\min C_{\alpha}$ designation which was shown previously to affect mass balance accuracy in the FD model.

FE solutions of organic liquid drainage and redistribution under gravity control have been shown to exhibit greater mass balance errors than corresponding infiltration problems. ${ }^{17}$ Therefore, mass balance behavior was examined in an advection dominated twophase NAPL drainage problem in system 2 using initial and boundary conditions given in Table 2. For the purpose of investigating mass balance accuracy only, these simulations assume $P_{c}\left(S_{n}\right)$ is non-hysteretic such that the drainage function can adequately represent imbibition processes. Predicted organic saturation distributions on 40 - and 120-element grids are shown in Fig. 11.

In the upper plot, the FD P-S and pressure-based solutions agree closely and exhibit a strong dependence 

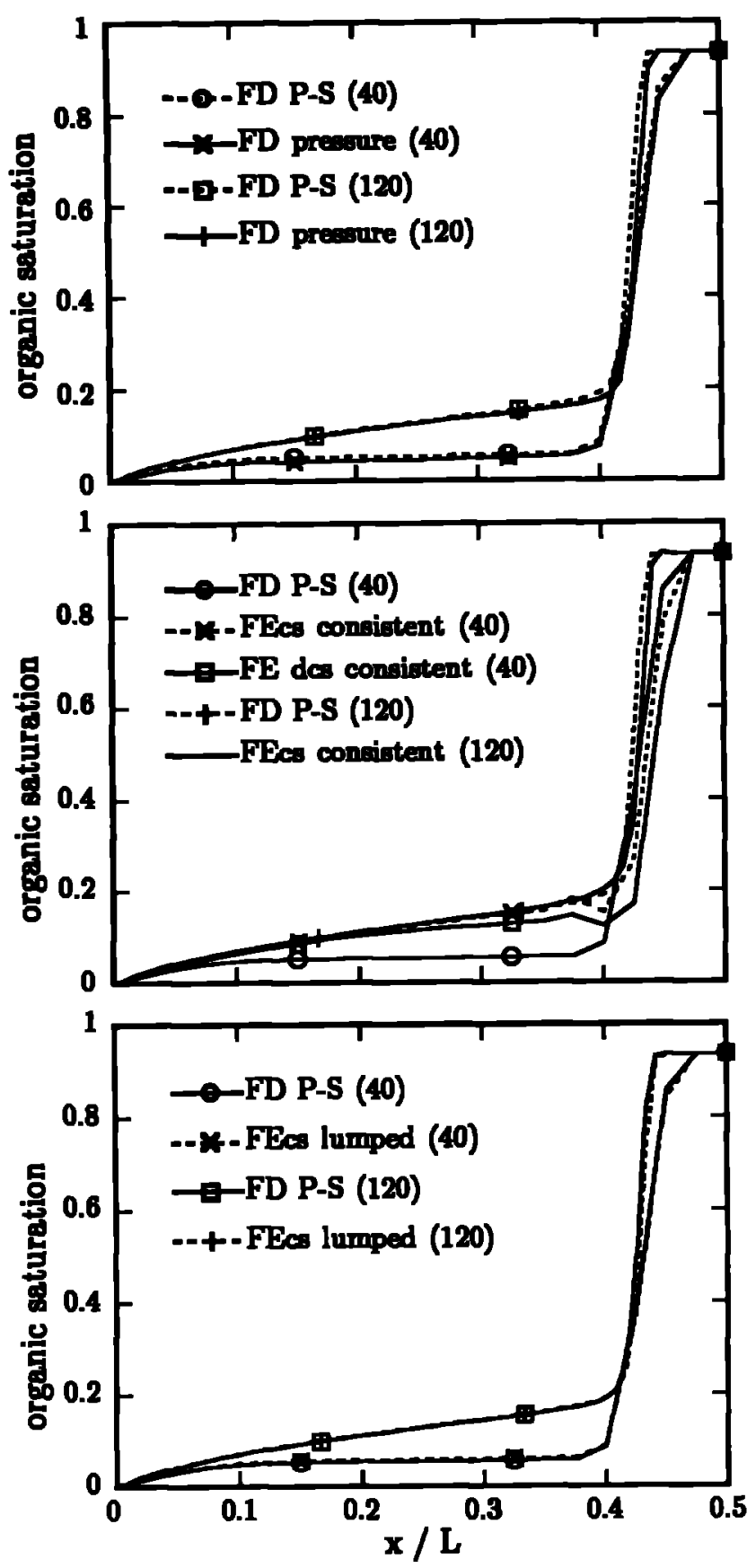

Fig. 11. Comparison of predicted NAPL saturation distributions for the organic liquid drainage problem at simulation time $=10 \mathrm{~s}$.

on mesh spacing. The coarse grid solutions exhibit a slightly dispersed drainage front and a saturation profile behind the front which differs markedly from that of the fine grid solutions. Although saturation profiles are not in agreement, both coarse and fine grid solutions produce good cumulative mass balance accuracy as shown in Table 5. This problem demonstrates mass conservation is not a sufficient condition for solution accuracy.

The observed discrepancies in the saturation profiles are believed to be due to the hyperbolic character of the coupled flow equations during drainage over the flat linear portion of $\boldsymbol{P}_{c}\left(S_{k}\right)$ where capillary dissipative effects are small. Analogous to the Buckley-Leverett problem, solving these equations with midpoint weighting on the transmissibility terms produces mass conservative, but physically inaccurate, solutions. ${ }^{6}$ It can be shown that additional numerical dissipation from upstream weighting of transmissibilities on the 40element grid produces solutions which are aligned closely with the midpoint weighting results on the 120element grid.

Inspection of the middle plot in Fig. 11 shows that FE solutions with consistent mass matrices do not exhibit the same spatial discretization dependence of the saturation profile behind the drainage front. The coarse grid FE solutions do show some numerical dispersion at the saturation front, similar to the FD solutions, and also a slight undershoot at the toe of the front, analogous to that observed with unsaturated flow models. ${ }^{7}$ The fine grid FE consistent and FD solutions agree very closely.

The effect of lumping is displayed in the bottom plot in Fig. 11. The lumped FEcs scheme is equivalent to the FD pressure-based formulation, and thus, organic saturation predictions exhibit similar behavior and close agreement with the FD results. Mass lumping eliminates undershoot at the toe of the front, but also creates a discretization dependence of the saturation profile observed with the FD solutions. The lumped dcs and rdcs schemes produced similar results, but are not shown in Fig. 11. Inspection of Table 5 shows mass lumping reduced the cumulative mass balance error, although this is an insufficient criterion for accuracy in this advection dominated problem; it also improved stability and reduced the computational work.

\section{CONCLLSIONS AND RECOMMENDATIONS}

This research has identified causes of mass balance errors observed in pressure-based two phase flow models. A number of remedies have been proposed and evaluated. The results demonstrate that mass conservative predictions can be obtained with FE and FD pressure-based or pressure-saturation formulations if capacity coefficients and initial conditions are properly specified. Specific conclusions and recommendations are summarized below:

1. When NAPL infiltration into a pristine aquifer is considered, mass balance accuracy depends on the proper specification of the initial nonexistent NAPL pressure distribution. Mass conservative solutions are obtained when the initial NAPL pressure distribution is established on the basis of $P_{c}=0$.

2. Capacity coefficients imbedded in the pressure- 
Table 5. Comparion of commlative mas halance erross and computaional wort from coneletent and mass hmped formulations in the NAPL drinage problem ing 40 and 120-elenent end

\begin{tabular}{|c|c|c|c|c|}
\hline \multirow[t]{2}{*}{$C_{\alpha}$ Form } & \multicolumn{2}{|c|}{ Consistent form } & \multicolumn{2}{|c|}{ Mass lumped form } \\
\hline & Cumulative error (\%) & Work $^{a}$ & Cumulative error (\%) & Worke \\
\hline $\begin{array}{l}\text { FD pressure based (40) } \\
\text { FD pressure based }(120) \\
\text { FD P-S formulation (40) } \\
\text { FD P-S formulation (120) } \\
\text { FEcs }(40) \\
\text { FEcs }(120) \\
\text { FEdcs }(40) \\
\text { FErdcs }(40)\end{array}$ & $\begin{array}{l}1 \cdot 7(-4) \\
1 \cdot 9(-4) \\
1 \cdot 2(-6) \\
1 \cdot 8(-6) \\
0 \cdot 20 \\
0 \cdot 28 \\
2 \cdot 48 \\
b\end{array}$ & $\begin{array}{l}5 \cdot 55 \\
5 \cdot 92 \\
4 \cdot 74 \\
5 \cdot 02 \\
5 \cdot 05 \\
5 \cdot 49 \\
7 \cdot 93\end{array}$ & $\begin{array}{l}0.07 \\
0.03 \\
1.07 \\
0.93\end{array}$ & $\begin{array}{l}4 \cdot 79 \\
5 \cdot 13 \\
4-88 \\
4-88\end{array}$ \\
\hline
\end{tabular}

Work $=\log$ (number of iteration $\times$ number of time steps).

${ }^{b}$ Would not converge.

based formulation can be made sufficiently small such that they do not appreciably affect mass balance accuracy or numerical solutions when $P_{c}=0$. In this work a min $C_{\alpha}=1(-6)$ was found to provide both good mass balance accuracy and good covergence performance. Examination of solution sensitivity to $\min C_{\alpha}$ is recommended to ensure acceptable mass balance results.

3. Mass balance errors in FE pressure-based models result from failure to preserve expansion of $\partial S / \partial t$ over the element when $P_{c}\left(S_{w}\right)$ is nonlinear. The scs and analytical $C_{\alpha}$ approximations employed in traditional FE pressure-based models do not necessarily preserve expansion of $\partial S / \partial t$ in the van Genuchten form of $P_{c}\left(S_{w}\right)$ over the nonlinear segment near $S_{w}=1$. An analysis of the residual error, $\epsilon^{*}$, shows that error canceling effects and improved mass balance accuracy can be obtained by increasing the explicitness of time weighting on $C_{a}$, together with proper selection of $\Delta t$. The residual error expressions can also be used to explain oscillatory behavior in the incremental mass balance error observed in all pressure-based models. When $P_{c}\left(S_{n}\right)$ is linear, $C_{\alpha}$ is constant yielding good mass balance accuracy in all models.

4. Conservation of mass in FE pressure-based solutions can be obtained when $C_{\alpha}$ is formulated to preserve the elemental approximation of the saturation time derivative. Within the traditional FE framework the expansion is preserved with the element-dependent dcs and rdcs approximations. In a non-traditional FE approach using fewer basis functions, expansion of the saturation derivative is preserved with the FEcs form.

Although superior to previous FE formulations, the dcs and rdcs approximations exhibit some instabilities manifested by large amplitudes in $\operatorname{MBE}(t)$, a smaller convergence range than corresponding FD and FEcs schemes, and cumulative mass balance errors which are good but generally greater than $1 \%$. These instabilities are apparently related to the distributed nature of the time derivative since they are virtually eliminated with mass lumping procedures. Additionally, the elemental dependence of the dcs and rdcs schemes complicates extensions to higher order interpolating functions or multi-dimensional systems. In comparison, the scs approximations used in the FEcs formulation are continuous at the element boundaries and therefore are easily extended. The FEcs scheme also has advantages in accuracy and stability over the des and $r d e s$ forms.

5. Mass lumping generally improves stability and mass balance accuracy of the dcs and rdcs FE schemes and eliminates undershoot behavior in coarse grid solutions. However, for advection dominated systems, FE schemes with consistent mass matrices are more accurate than lumped schemes at a given discretization. Coarse grid, lumped scheme solutions for such systems exhibited an incorrect distribution of saturations behind the drainage front.

Mass lumping in the FEcs formulation was not beneficial in NAPL infiltration problems and only slightly reduced cumulative error and work requirements in advection dominated organic drainage problems. Thus, there appears to be no clear advantage in the application of mass lumping to the FEcs scheme.

6. The consistent FEcs formulation is recommended for FE pressure-based multiphase flow models on the basis of superior accuracy, stability, and ease in implementation.

\section{ACKYOWLEDGEMENTS}

This work was supported in part by the National Science Foundation under grant EID-9023090 and the Electric Power Research Institute under contract RP 28798. The paper has not been subject to NSF or EPRI review, and thus the views and opinions herein do not 
necessarily reflect those of the NSF or EPRI. We gratefully acknowledge Howard Reeves for his insight and discussions in the development of this work and for guidance in using his model.

\section{REFERENCES}

1. Abriola, L.M., Multiphase migration of organic compounds in a porous medium. A Mathematical Model. In Lecture Notes in Engineering, ed. C.A. Brebbia \& S.A. Orszag. Springer-Verlag, Berlin, 1984.

2. Abriola, L.M., Modeling multiphase migration of organic chemicals in groundwater systems - A review and assessment. Environ. Health Perspectives, 83 (1989) 11743.

3. Abriola, L.M. Pinder, G.F., A multiphase approach to the modeling of porous media contamination by organic compounds 1. Equation development. Water Resour. Res., 21 (1), (1985) 11-18.

4. Abriola, L.M. \& Pinder, G.F., A multiphase approach to the modeling of porous media contamination by organic compounds 2. Numerical simulation. Water Resour. Res., 2 (1) (1985) 19-26.

5. Allen, M.B., Numerical modeling of multiphase flow in porous media. In Advances in Transport Phenomena in Porous Media, ed. J. Bear \& M.Y. Corapcioglu Martinus Nijhoff Publishers, Dordrecht, 1987, pp. 851-920.

6. Aziz, K. \& Settari, A., Petrolewn Reservoir Simulation. Applied Science Publishers, London, 1979.

7. Celia, M.A., Boulouton, E.T. \& Zarba, R.L., A general mass-conservation numerical solution for the unsaturated flow equation. Water Resource. Res., 26 (7) (1990) 148396.

8. Demond, A.H. \& Roberts, P.V., Effect of interfacial forces on two-phase capillary pressure-saturation relationships. Water Resour. Res., 27 (3) (1991) 423-38.

9. Faust, C.R., Transport of immiscible fluids within and below the unsaturated zone: A numerical model. Water Resour. Res., 21 (4) (1985) 587-96.

10. Faust, C.R., Guswa J.H. \& Mercer, J.W., Simulation of three-dimensional flow of immiscible fluids within and below the unsaturated zone. Water Resour. Res., 25 (12) (1989) 2449-64.

11. Forsyth, P.A., Simulation of nonaqueous phase groundwater contamination. Adv. Water Resour., 11 (1988) 7483.

12. Forsyth, P.A. \& Shao, B.Y., Numerical simulation of gas venting for NAPL site remediation. Adv. Water Resour., 14 (6) (1991) 354-67.

13. Gamliel, A., Simulation of Immiscible Multiphase Flow in Porous Media using a Moving Finite Element Algorithm PhD Dissertation, Lniversity of Michigan, Ann Arbor, MI, 1989.
14. Gamliel, A. \& Abriola, L.M., A one-dimensional moving grid solution for the coupled non-linear equations governing multiphase flow in porous media. 1: model development. Int. J. for Numerical Methods in Fluids, 14 (1992) 25-45.

15. Høst-Madsen, J., Immiscible multi-phase flow in porous media, Institute of Hydrodynamics and Hydraulic Engineering, Series Paper 49, Technical Lniversity of Denmark, Lyngby, 1989.

16. Huyakorn, P.S. \& Pinder, G.F., Computational Methods in Subsurface Flow. Academic Press, San Diego, 1983.

17. Kaluarachchi, J.J. \& Parker, J.C., An efficient finite element method for modeling multiphase flow. Water Resour. Res., 25 (1) (1989) 43-54.

18. Kueper, B.H., Abbott, W. \& Farquhar, G., Experimental observations of multiphase flow in heterogeneous porous media. J. of Contominant Hydrology, 5 (1989) 83-95.

19. Kueper, B.H. \& Frind, E.O., Two-phase flow in heterogeneous porous media, 1. Model development. Water Resour. Res., 27 (6) (1991) 1049-57.

20. Kuppusamy, T., Sheng, J., Parker, J.C. \& Lenhard, J., Finite-element analysis of multiphase immiscible flow through soils. Water Resour. Res., 23 (4) (1987) 625-31.

21. McWhorter, D.N. \& Sunada, D.K., Exact integral solutions for two-phase flow. Water Resour. Res., 26 (3) (1990) 399-414.

22. Milly, P.C.D., A mass-conservative procedure for timestepping in models of unsaturated flow. Adv. Water Resour., 8 (1985) 32-6.

23. Milly, P.C.D., Advances in modeling of water in the unsaturated zone. Transport in Porous Media, 3 (1988) 491-514.

24. Osborne, M. \& Sykes, J., Numerical modeling of immiscible organic transport at the Hyde Park landfill. Water Resour. Res., 22 (1) (1986) 25-33.

25. Parker, J.C., Multiphase flow and transport in porous media. Reviews of Geophysics, 27 (3) (1989) 311-28.

26. Reeves, H.W., Volatilization and Vapor Phase Transport of Organic Contaminants in the Subsurface PhD Dissertation, University of Michigan, Ann Arbor, MI, 1991.

27. Reeves, H. \& Abriola, L.M., A decoupled approach to the simulation of flow and transport on non-aqueous organic phase contaminants through porous media. In Developments in Water Science, ed. M.A. Celia, L.A. Ferrand,C.A. Brebbia, W.G. Gray \& G.F. Pinder. Elsevier, Amsterdam, 1988, pp. 147-52.

28. Reeves, H. \& Abriola, L.M. An iterative-compositional model for subsurface multi-phase flow. (submitted) J. of Contaminant Hydrology.

29. van Genuchten, M.T., A closed-form equation for predicting the hydraulic conductivity of unsaturated soils. Sci. Soc. Am. J., 44 (1980) 892-8.

30. Zienkiewicz, O.C. \& Taylor, R.L., The Finite Element Method. McGraw-Hill, London, 1991. 